\title{
CHANDRA VIEW OF THE WARM-HOT INTERGALACTIC MEDIUM TOWARD 1ES 1553+113: ABSORPTION-LINE DETECTIONS AND IDENTIFICATIONS. I.
}

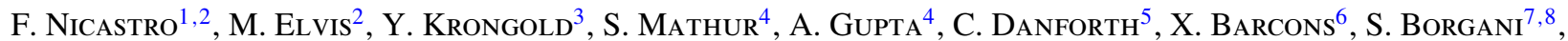 \\ E. Branchini ${ }^{9}$, R. Cen $^{10}$, R. Davé ${ }^{11}$, J. KaAstra ${ }^{12}$, F. Paerels ${ }^{13}$, L. Piro ${ }^{14}$, J. M. Shull ${ }^{5}$, Y. Takei ${ }^{15}$, and L. Zappacosta ${ }^{1}$ \\ ${ }^{1}$ Osservatorio Astronomico di Roma-INAF, Via di Frascati 33, I-00040 Monte Porzio Catone, RM, Italy \\ ${ }^{2}$ Harvard-Smithsonian Center for Astrophysics, 60 Garden St., MS-04, Cambridge, MA 02138, USA \\ ${ }^{3}$ Instituto de Astronomia, Universidad Nacional Autonoma de Mexico, Mexico City Mexico \\ ${ }^{4}$ Ohio State University, Columbus, OH, USA \\ ${ }^{5}$ CASA, Department of Astrophysical and Planetary Sciences, University of Colorado, 389-UCB, Boulder, CO 80309, USA \\ ${ }^{6}$ Instituto de Física de Cantabria (CSIC-UC), E-39005 Santander, Spain \\ ${ }^{7}$ Dipartimento di Astronomia dell’Università di Trieste, Via G.B. Tiepolo 11, I-34131 Trieste, Italy \\ ${ }^{8}$ INAF- Osservatorio Astronomico di Trieste, Via G.B. Tiepolo 11, I-34131 Trieste, Italy \\ 9 Dipartimento di Fisica “E. Amaldi," Università degli Studi “Roma Tre,” via della Vasca Navale 84, I-00146 Roma, Italy \\ ${ }^{10}$ Princeton University Observatory, Princeton, NJ 08544, USA \\ ${ }^{11}$ Astronomy Department, University of Arizona, Tucson, AZ 85721, USA \\ 12 SRON Netherlands Institute for Space Research, Sorbonnelaan 2, 3584 CA, Utrecht, The Netherlands \\ ${ }^{13}$ Columbia Astrophysics Laboratory and Department of Astronomy, Columbia University, 550 West 120th Street, New York, NY 10027, USA \\ ${ }_{14}^{14}$ INAF-IAPS, Via del Fosso del Cavaliere 100, I-00133 Roma, Italy \\ ${ }^{15}$ Institute of Space and Astronautical Science, Japan Aerospace Exploration Agency, 3-1-1 Yoshinodai, Chuo-ku, Sagamihara, Kanagawa 252-5210, Japan \\ Received 2012 October 26; accepted 2013 March 12; published 2013 May 7
}

\begin{abstract}
We present the first results from our pilot $500 \mathrm{ks}$ Chandra Low Energy Transmission Grating Large Program observation of the soft X-ray brightest source in the $z \gtrsim 0.4$ sky, the blazar 1ES $1553+113$, aimed to secure the first uncontroversial detections of the missing baryons in the X-rays. We identify a total of 11 possible absorption lines, with single-line statistical significances between $2.2 \sigma$ and 4.1 $\sigma$. Six of these lines are detected at high single-line statistical significance $(3.6 \leqslant \sigma \leqslant 4.1)$, while the remaining five are regarded as marginal detections in association with either other X-ray lines detected at higher significance and/or far-ultraviolet (FUV) signposts. Three of these lines are consistent with metal absorption at $z \simeq 0$, and we identify them with Galactic O I and C II. The remaining eight lines may be imprinted by intervening absorbers and are all consistent with being high-ionization counterparts of FUV $\mathrm{H}_{\mathrm{I}}$ and/or O VI intergalactic medium signposts. In particular, five of these eight possible intervening absorption lines (single-line statistical significances of $4.1 \sigma, 4.1 \sigma, 3.9 \sigma, 3.8 \sigma$, and $2.7 \sigma$ ), are identified as $\mathrm{C} \mathrm{V}$ and $\mathrm{C}$ VI $\mathrm{K} \alpha$ absorbers belonging to three WHIM systems at $z_{X}=0.312, z_{X}=0.237$, and $\left\langle z_{X}\right\rangle=0.133$, which also produce broad Hi (and O VI for the $z_{X}=0.312$ system) absorption in the FUV. For two of these systems $\left(z_{X}=0.312\right.$ and 0.237$)$, the Chandra X-ray data led the a posteriori discovery of physically consistent broad $\mathrm{H}_{\mathrm{I}}$ associations in the FUV (for the third system the opposite applies), so confirming the power of the X-ray-FUV synergy for WHIM studies. The true statistical significances of these three X-ray absorption systems, after properly accounting for the number of redshift trials, are $5.8 \sigma\left(z_{X}=0.312 ; 6.3 \sigma\right.$ if the low-significance $\mathrm{O} v$ and $\mathrm{C} \mathrm{v} \mathrm{K} \beta$ associations are considered), $3.9 \sigma\left(z_{X}=0.237\right)$, and $3.8 \sigma\left(\left\langle z_{X}\right\rangle=0.133\right)$, respectively.
\end{abstract}

Key words: atomic processes - intergalactic medium - line: identification - opacity - X-rays: general

Online-only material: color figures

\section{INTRODUCTION}

According to the latest baryon census in the local $(z \lesssim 0.4)$ universe (Shull et al. 2012, and references therein), about $30 \%-40 \%$ of the total number of baryons predicted by big bang nucleosynthesis (e.g., Kirkman et al. 2003), inferred by density fluctuations of the cosmic microwave background (e.g., Bennett et al. 2003; Spergel et al. 2007), and seen at $z \sim 3$ in the "Ly $\alpha$ Forest" (e.g., Rauch 1998; Weinberg et al. 1997), are missing at the present epoch.

Of the low- $z$ baryons we can account for in a recent census (Shull et al. 2012), $(18 \pm 4) \%$ lie in a collapsed phase (stars, cold atomic and molecular gas in galaxies, circumgalactic medium (CGM), and hot virialized gas in groups and clusters of galaxies), (28 \pm 11$) \%$ lie in the local photoionized Ly $\alpha$ forest, and finally, as much as $(25 \pm 8) \%$, are estimated to be found in the $\mathrm{O} v \mathrm{vI}$ BLA-traced warm-hot intergalactic medium (IGM). ${ }^{16}$ The remaining ( $29 \pm 13) \%$ of baryons still remains elusive, and are likely to lie in the hot phase of the WHIM, at temperatures larger than $\log T \simeq 5.5 \mathrm{~K}$.

This is predicted by nearly all hydrodynamical simulations for the formation of structures in a $\Lambda \mathrm{CDM}$ universe (e.g., Cen \& Ostriker 2006; Tornatore et al. 2010; Bertone et al. 2010; Branchini et al. 2009; Smith et al. 2011). According to simulations, starting at redshift of $z \sim 2$ some of the

\footnotetext{
16 This estimate is based on an average ionization and metallicity, versus column density, correction derived by hydrodynamical simulations, and therefore suffers not only the large scatter found in the given simulation between these parameters (i.e., Figure 8 in Shull et al. 2012), but also the scatter of theoretical predictions from simulation to simulation (e.g., Cen \& Ostriker 2006; Tornatore et al. 2010; Bertone et al. 2010; Branchini et al. 2009; Smith et al. 2011). The resulting number of baryons still missing also depends on these uncertainties.
} 
IGM was shock-heated to temperatures of $10^{5}-10^{7} \mathrm{~K}$ during the continued process of collapse and structure formation, and enriched up to $Z_{\text {WHIM }}=0.1-1 Z_{\odot}$ by galaxy super-winds (e.g., Cen $\&$ Ostriker 2006) already at redshifts of $\sim 0.5$, remaining essentially unchanged from then to $z \simeq 0$. The first pieces of observational evidence of this violent process of structure formation are starting to be gathered in the densest nodes of this filamentary IGM, at and inside the virial radius of massive clusters of galaxies (e.g., Keshet et al. 2012). However, diffuse WHIM filaments are too tenuous to be detected through their bremsstrahlung and line emission with current far-ultraviolet (FUV) or X-ray instruments (e.g., Yoshikawa et al. 2003). Absorption is more promising.

The lowest temperature tail of the WHIM distribution, from $T \sim 10^{5}$ up to $\sim 10^{5.5} \mathrm{~K}$ (where the fraction of $\mathrm{O}$ VI peaks), has been most likely detected in the FUV, with FUSE and the STIS spectrometer on board the Hubble Space Telescope $(H S T)$, in O VI and narrow Ly $\alpha$ absorbers, though the fraction of this gas that actually belongs to the shock-heated WHIM is highly uncertain (e.g., Danforth \& Shull 2008). More recently, the advent of the larger throughput Cosmic Origins Spectrograph (COS) of HST has enabled the detection of somewhat hotter $\left(T \sim 10^{5}-10^{5.7}\right)$ and possibly truly shock-heated WHIM, through the detectability of the strongest (i.e., large column density) broad Ly $\alpha$ absorbers (BLAs) with thermal Doppler parameter $b_{\text {th }}=\sqrt{2 k T / m_{p}} \simeq 40-90 \mathrm{~km} \mathrm{~s}^{-1}$ (Danforth et al. $2010 \mathrm{~b}) .{ }^{17}$ These BLAs are in principle good tracers of the warm $\left(T \sim 10^{5}-10^{5.7} \mathrm{~K}\right)$ portion of the WHIM, where a significant fraction of the WHIM ( 30\%-40\%; e.g., Cen \& Ostriker 2006) is supposed to reside. However, observing $b_{\text {th }} \simeq 40-90 \mathrm{~km} \mathrm{~s}^{-1}$ BLAs requires data of excellent quality and accurate continuum definition to detect the low-contrast features. In addition, measuring $\mathrm{H}_{\mathrm{I}}$ without an ionization correction ${ }^{18}$ will always underestimate the actual baryon fraction in the fraction of hot IGM sampled by these tracers. Given the temperature of this gas, such an ionization correction can only come from the X-ray detection of the highly ionized counterparts to such BLAs. Finally, the $T \gtrsim 10^{5.7} \mathrm{~K}$ portion of the WHIM (the vast majority of the WHIM mass) can only be detected through highly ionized metals in the X-rays.

However, detecting the metal tracers of the bulk of the "missing baryons" in the "X-ray Forest," has proven to be extremely difficult. This is because of the unfortunate combination of (1) the still limited resolving power $(R \sim 400$ at the location of the $\mathrm{O}$ VII $\mathrm{K} \alpha$ transition, at $\lambda=21.6 \AA$ ) and the low throughput $\left(A_{\mathrm{Eff}} \sim 15-70 \mathrm{~cm}^{2}\right)$ of the current high-resolution X-ray spectrometers (the XMM-Newton Reflection Grating Spectrometer (RGS; den Herder et al. 2001) and the Chandra Low Energy Transmission Grating (LETG; Brinkman et al. 2000)), (2) the lack of bright $\left(f_{0.5-2 \mathrm{keV}} \geqslant 10^{-11} \mathrm{erg} \mathrm{s}^{-1} \mathrm{~cm}^{-2}\right)$ extragalactic point-like targets at sufficiently high redshifts, and (3) the dramatic steepening in slope $(\Delta \alpha \gtrsim 1.5)$ of the predicted number density of metal He-like WHIM filaments per unit redshift at ion column densities $\gtrsim 10^{15} \mathrm{~cm}^{-2}$ (e.g., Cen \& Fang 2006). Current evidence is still limited, and highly controversial.

\footnotetext{
17 The thermal Doppler parameter, is typically narrower than the measured Doppler parameter $b$, which includes a combination of internal turbulence and Hubble-flow broadening. Danforth et al. (2010b) estimate an average correction factor $\left(b / b_{\text {th }}\right) \simeq 1.2$.

18 The measured H I Doppler parameter gives only an upper limit on the gas temperature, and in addition, the proper ionization correction depends also on the gas density because of the second-order contribution of photoionization, and this can only be estimated through multi-ion metal measurements, e.g., Nicastro et al. (2002).
}

The statistically strongest evidence to date comes from the evidence for large amounts of baryonic matter in two intervening WHIM filaments along the line of sight to the nearby $(z=0.03)$ blazar Mkn 421 (Nicastro et al. 2005a, 2005b). However, these results are controversial (Kaastra et al. 2006; Rasmussen et al. 2007; Danforth et al. 2011; Yao et al. 2012; but see also Williams et al. 2006, 2010; Nicastro et al. 2008).

Over the last few years there have been new discoveries reported (Buote et al. 2009: B09; Fang et al. 2010: F10; Nicastro et al. 2010; Zappacosta et al. 2010, 2012). However, such tentative discoveries are either serendipitous (and so biased toward the strongest systems; Nicastro et al. 2010; Zappacosta et al. 2012), or trace extreme galaxy overdensity regions (Zappacosta et al. 2010) or even just galaxy halos (Buote et al. 2009; Zappacosta et al. 2010; Williams et al. 2012). In all cases, these sample only the hottest and densest IGM or CGM, which is not representative of the majority of the WHIM.

Thus a firm, high-significance detection of the bulk of the missing baryon mass has not been secured yet. To do so, the only pursuable observational strategy, with current instrumentation, is to first identify "true" gaseous signposts (e.g., Mathur et al. 2003) of the $T \sim 10^{5.3}-10^{5.7}$ WHIM (i.e., BLAs), and then search for their metal counterparts in the soft X-rays, along optimally selected sightlines. Over the past two years we selected 1ES $1553+113$ as "the best WHIM target in the universe" (because of its extreme soft X-ray brightness, relatively high redshift, and the presence of BLA signposts in its HST-COS spectrum-Danforth et al. 2010a), and started the X-ray campaign with a pilot $500 \mathrm{ks}$ Chandra cycle 12 observation of 1ES $1553+113$. In our observing proposal, we conservatively tuned the exposure of this first pilot observation to test the reliability of the strongest FUV systems as gaseous signposts for X-ray WHIM filaments. The results are well beyond the most optimistic expectations and confirm the effectiveness of such an observational strategy. In this article, we report on these results from the pilot Chandra-LETG spectrum.

The paper is organized as follows. In Section 2, we describe the target of our LETG observation, and summarize the relevant results from the HST-COS observations reported by Danforth et al. (2010a). Section 3 describes the Chandra-LETG data reduction and analysis, while in Section 4, we discuss our findings and present possible absorption system identifications. Finally in Section 5, we summarize our conclusions. In a forthcoming article ("Paper II"), we will present a self-consistent modeling of the spectral features detected in the LETG spectrum of $1 E S 1553+113$ by making use of our WHIM hybrid collisional-ionization plus photoionization models, and so deriving preliminary estimates of metallicities of these systems, the ionization-corrected baryon cosmological mass density of the WHIM at $T \sim 10^{5.5} \mathrm{~K}$, and the number density of $\mathrm{C} \mathrm{V}$ and O VII WHIM absorbers as a function of the ion column density.

\section{1ES 1553+113 AND THE WHIM SIGNPOSTS ALONG ITS LINE OF SIGHT}

Four years ago, we selected 1ES $1553+113$ as the optimal target for FUV and X-ray WHIM studies, because of its relatively high estimated redshift prior to spectroscopic determinations $(z \gtrsim 0.25$, from the non-detection of the host galaxy in a deep HST R image; Treves et al. 2007), its high Galactic latitude ( $b \simeq 44^{\circ}$ ), and its extreme average $0.1-2.4 \mathrm{keV}$ brightness of $2 \mathrm{mCrab}\left(4 \times 10^{-11} \mathrm{erg} \mathrm{s}^{-1} \mathrm{~cm}^{-2}\right)$, coupled with a bright and stable FUV flux of $v f_{v}(1600 \AA) \simeq 3 \times 10^{-11} \mathrm{erg} \mathrm{s}^{-1} \mathrm{~cm}^{-2}$. 
1ES 1553+113 was first observed with the HST-COS on 2009 September 22, for three orbits (one orbit with the G130M and two with the G160M grating; Danforth et al. 2010a). Almost 2 yr later, on 2011 July 24, the target was re-observed for two additional orbits with the G130 M and four additional orbits with the G160M. The first COS spectrum of 1 ES $1553+113$ was published in 2010 by Danforth and collaborators. Here, we summarize the main findings from this 2009 observation, relevant to this work.

The published HST-COS spectrum of 1ES 1553+113 secured a robust lower limit on the spectroscopic redshift of $1 \mathrm{ES}$ 1553+113. Narrow H I Ly $\alpha$ (NLA) and O VI absorption is observed at a combined $19.3 \sigma$ significance at $z=0.395$ (Figure 2 of Danforth et al. 2010a), establishing a spectroscopic lower limit on the redshift of 1ES $1553+113$ of $z \gtrsim 0.4$ (almost twice the non-spectroscopic value upon which we based our original selection) and making this target by far the brightest, non-transient, $X$-ray source in the $z \geqslant 0.4$ sky. Moreover, Danforth et al. (2010a) report on the presence of five possible BLAs, at $z=0.04281,0.10230,0.12325,0.13334$, and 0.15234 , plus a rare and complex triple-H I-metal absorption system at $z=0.1864-0.1899$, spanning a range of $1200 \mathrm{~km} \mathrm{~s}^{-1}$ in velocity (Figure 2 and Table 1 in Danforth et al. 2010a). In our work, we use these systems as initial gaseous signposts for our X-ray search of either WHIM filaments (BLA counterparts), or highly ionized galaxy outflows and hot CGM from nearby galaxies (counterparts to the triple-H I-metal system). However, since the 2011 COS observation almost doubled the signalto-noise ratio $(\mathrm{S} / \mathrm{N})$ of the first published spectrum of $1 \mathrm{ES}$ $1553+113$, we also retrieved and reduced the total final COS spectrum of 1ES $1553+113$, and present here a re-analysis of it whenever the higher $\mathrm{S} / \mathrm{N}$ data detect FUV lines associated to the proposed X-ray identifications and that had eluded detections in the published spectrum because of its lower $\mathrm{S} / \mathrm{N}$.

\section{THE CHANDRA-LETG SPECTRUM OF 1ES 1553+113}

Chandra observed 1ES 1553+113 between 2011 May and June, for $500 \mathrm{ks}$, with the LETG spectrometer (constant resolution $\Delta \lambda=50 \mathrm{~m} \AA$, i.e., resolving power $R=400$ at $\lambda=20 \AA$ ) dispersed across the High Resolution Camera for Spectroscopy (HRC-S; Murray \& Chappell 1996) plates. The observation consisted of three visits with exposures of $166.3 \mathrm{ks}$ (2011 May 4-6), 175.4 ks (2011 May 6-9), and 153.9 ks (2011 June 18-20).

\subsection{Data Reduction}

The data were reduced with the latest version of the Chandra Interactive Analysis of Observation software (CIAO v. 4.4.1, CALDB v. 4.5.2), following the standard processing procedures outlined in the HRC-S-LETG grating analysis thread. ${ }^{19}$

The Chandra HRC-S detector has virtually no spectral resolution, which prevents the separation of the different spectral orders dispersed by the LETG. For each observation, we therefore extracted all-order negative and positive sources and background spectra with the CIAO tool tg_extract. This produced a total of six LETG spectra, three (one per LETG visit) negativeorder and three positive-order source and background spectra, extracted at a binning pace of $\Delta \lambda=12.5 \mathrm{~m} \AA$, i.e., oversampling the LETG FWHM resolution of $50 \mathrm{m \AA}$ by a factor of four.

Due to the impossibility of separating HRC-LETG spectral orders, the spectral modeling of these spectra must be performed

\footnotetext{
19 http://cxc.harvard.edu/ciao/guides/gspec_hrcsletg.html
}

by folding the fitting models with the sum of the convolution products of the redistribution matrices (RMFs) and ancillary response files (ARFs) belonging to the first $N$ positive and negative orders, respectively. For our LETG observations of 1ES $1553+113$, we conservatively decided to build our final all-order response matrix by adding up positive and negative orders up to $N=10$. For each order, we used the CIAO tools mkgarf and mkgrmf to build 20 effective area and photonredistribution matrices, which were then read together with the data into the CIAO fitting-package Sherpa, through the commands load_multi_arfs and load_multi_rmfs.

To maximize the $\mathrm{S} / \mathrm{N}$ of our spectra (we note that the HRC-S-LETG combination has an effective area of only $A_{\text {Eff }} \simeq 15 \mathrm{~cm}^{2}$ at both $\lambda=22$ and $44 \AA$ ), we first coadded the three negative- and three positive-order spectra (source and background) from the three observations (CIAO tool add_grating_spectra), to produce two LETG spectra (one negative- and one positive-order spectrum), with net exposures of $495.6 \mathrm{ks}$ each. Effective area matrices of the three observations, for each of the ten negative and ten positive orders, were averaged together, producing 20 averaged effective area matrices (ARFs). Finally, we used the CIAO tool add_grating_orders to co-add the two negative- and positive-order source and background spectra and ARFs, to end up with a single LETG spectrum, containing all counts from the ten negative-order and the ten positive-order spectra of the three Chandra observations of $1 E S 1553+113$. The final co-added spectrum has roughly 250-300 net (i.e., background subtracted) counts per $\Delta \lambda=50 \mathrm{~m} \AA$, resolution element, over the two interesting spectral bands covering the $z<0.4$ O VII K $\alpha(\lambda=21.6-30 \AA)$ and $\mathrm{C} \mathrm{v} \mathrm{K \alpha}(\lambda=40.2-56 \AA)$ ranges at redshifts where WHIM absorption might be found, for a signal-to-noise ratio per resolution element $(\mathrm{S} / \mathrm{N}) \simeq 16$.

Because of our co-adding procedure, particular care must be taken to account for the wavelength calibration inaccuracy. The Chandra LETG-HRC-S spectrometer suffers a variable intrinsic wavelength uncertainty owing to the nonlinearity in the effective dispersion relation. This nonlinearity is due to a spatial imaging nonlinearity of the HRC-S detector caused by the combination of (1) partial charge loss in the 3-tap readout algorithm of each of the three HRC-S microchannel plates, each of which is divided into 190 taps along the dispersion direction; and (2) a hardware problem that corrupts the data from the position taps under a specific set of conditions. The net result is that photon event positions obtained from processing HRC-S telemetry can be offset from their true values. ${ }^{20}$ The wavelength errors thus depend on the physical location of dispersed photon events on the HRC-S, and so on both precise nominal pointing direction and the deliberate Lissajous figure "dither" about this point. ${ }^{21}$ An updated version of the degap polynomial coefficients based on empirical wavelength corrections from multiple HRC-S/ LETG observations of Capella has been released since CALDB 3.2.15. This update allows the correction of the nonlinearities in the HRC-S dispersion relation improving the $90 \%$ statistical significance uncertainties across the detector (i.e., $1.6 \sigma$ ) from $22 \mathrm{~m} \AA$ to $16 \mathrm{~mA}$. Since we have co-added three different observations, and then the resulting positive and negative orders, we should consider a larger uncertainty. By propagating four times, in quadrature, the quoted deviations, we obtain $90 \%$ uncertainties of $32 \mathrm{~m} \AA$. However, since the updated wavelength

\footnotetext{
20 See http://cxc.harvard.edu/cal/Letg/Hrc_disp/degap.html.

21 See, e.g., http://cxc.harvard.edu/cal/Letg/Corrlam/.
} 
corrections have been applied to the rest-frame position of the strongest soft X-ray metal electronic transitions and the interpolation of the correction may not be strictly valid to the position of blueshifted and redshifted lines, we conservatively adopt a $90 \%$ wavelength uncertainty $40 \mathrm{~m} \AA, 80 \%$ of the LETG line-spread function (LSF) FWHM.

In the following, we report all uncertainties at the $1 \sigma$ (68\%) statistical significance level, with the exception of X-ray wavelength and redshifts, for which we use $90 \%$ statistical confidences (i.e., 1.6 $\sigma$ ).

\subsection{Data Analysis}

We used the fitting package Sherpa, in CIAO, to fit our Chandra-LETG spectrum of 1ES $1553+113$. We ignored data outside the 10-60 $\AA$ spectral interval, where $\mathrm{S} / \mathrm{N}$ was either too low $(\lambda>60 \AA$ and $\lambda<5 \AA)$ and/or the spectral resolving power too low (at $\lambda<10 \AA, R<200$, implying $\Delta v>1500 \mathrm{~km} \mathrm{~s}^{-1}$ ).

We first fitted the spectrum with a model consisting of a single power law absorbed by neutral gas. This yielded a best-fitting power-law energy spectral index and cold-absorber column density of $\Gamma=2.66 \pm 0.01$ and $N_{\mathrm{H}}=(3.71 \pm 0.05) \times 10^{20} \mathrm{~cm}^{-2}$ (fully consistent with the Galactic value along the line of sight to $1 \mathrm{ES} 1553+113: N_{\mathrm{H}}^{\mathrm{Gal}}=3.65 \times 10^{20} \mathrm{~cm}^{-2}$; Kalberla et al. 2005). The integrated reduced $\chi^{2}$ was acceptable, $\chi_{r}^{2}($ dof $)=$ $0.90(3995)$. However, a visual inspection of the residuals showed both broad deviations around the $\mathrm{K}$ instrumental edges of $\mathrm{O} \mathrm{I}(\lambda=23.3 \AA)$ and $\mathrm{C} \mathrm{I}(\lambda=43.6 \AA)$, the Medges of I and $\mathrm{Cs}$ $(\lambda \simeq 11.5-12.4 \AA)$, and the HRC-S plate gaps $(\lambda \simeq 48-58 \AA)$, as well as narrow deficit of counts at specific wavelengths. Figure 1 (top panel) shows these residuals smoothed through a convolution with the LETG LSF, in re-normalized (after smoothing) standard deviations.

At first, we excluded the possible absorption lines (which do not affect the continuum fitting over the integrated $10-60 \AA$ band) and focused on curing the residual calibration uncertainties around the positions of the instrumental $\mathrm{I}, \mathrm{Cs}, \mathrm{O}$, and $\mathrm{C}$ edges and the HRC-S plate gaps, with the aim of reaching an optimal model of the continuum that would allow us to properly assess the statistical significance of true physical spectral features possibly present in the spectrum. We then added to the continuum model (1) an edge with negative optical depth, to cure the sharp edge-like emission feature present in the residuals at $\lambda \simeq 43.8 \AA$ and due to either an overestimated modeling of the $C_{I}$ instrumental edge in the LETG effective area, or a nonaccurate modeling of the $\mathrm{C}$ I absorption edge in the wabs model of XSPEC, or both (best-fit $\tau=-0.04$ ); (2) three broad emission Gaussians at $\lambda=41.61 \AA, \lambda=50.10 \AA$, and $\lambda=56.39 \AA$ (best-fitting $\sigma=0.8 \AA$ for the first two and $\sigma=0.2 \AA$ for the third) to model residuals broad wiggles at the wavelength of the $\mathrm{C}$ I edge, and the dithering-shaped HRC-S plate gaps ${ }^{22}$; (3) five notch XSPEC component models: four negative (at $\lambda=11.48$, $43.99,48.34$, and $52.14 \AA$, with total widths of $0.6,0.4,0.2$, and $1.1 \mathrm{~A}$, respectively), to model residual narrower (but still well resolved) deviations at the I and $\mathrm{Cs} \mathrm{M}$ edges and the $\mathrm{C} \mathrm{K}$ edge and plate-gap wavelengths (Figure 1, top panel), and one positive with central $\lambda=20.14 \AA$ and total width of $5.5 \AA$, to

\footnotetext{
22 During every pointing the Chandra satellite dithers around the aim-point sky coordinates producing a Lissajous figure in detector coordinates. This implies that plate gaps are not blind to photons at all times during the observation, and their dispersion position (for dispersed spectra) can be reconstructed a posteriori by applying the aspect solution. However, the exact shape of the effective area at the plate-gap wavelengths depends on the accuracy of the aspect reconstruction and the zeroth-order centroid.
}

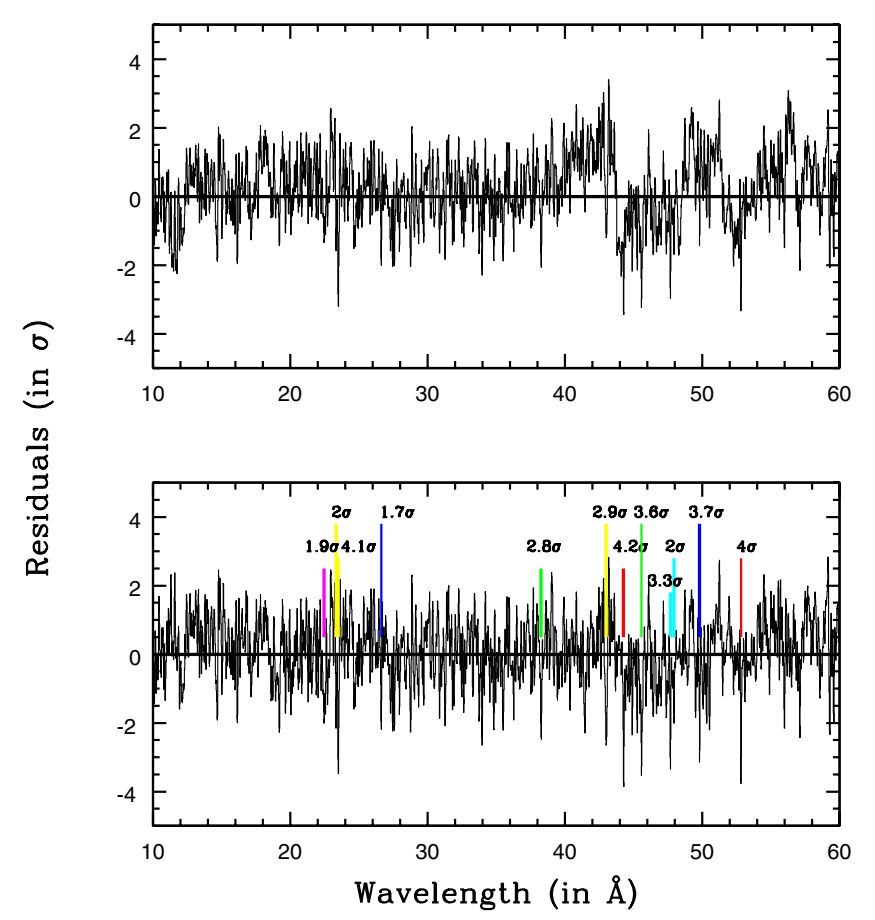

Figure 1. LSF-smoothed residuals (in re-normalized, after smoothing, standard deviations) of the 10-60 500 ks LETG spectrum of 1 ES $1553+113$, to (a) a model consisting of a power law absorbed by a neutral absorber (top panel), and (b) the same continuum model with the addition of seven components (an edge with negative optical depth, two broad Gaussians, three negative, and one positive notches, to cure the residual systematic calibration uncertainties visible in the top panel and due to the instrumental $\mathrm{O}_{\mathrm{I}}$ and $\mathrm{C}$ I edges and the ditheringshaped HRC-S-LETG effective area at the wavelengths of the HRC-S plate gaps (bottom panel). In the bottom panel, the positions of the 12 possible absorption lines are marked with color-coded segments (Section 4) and labeled with their single-line statistical significances.

(A color version of this figure is available in the online journal.)

model a systematic excess over the 18-23 Å spectral region, just leftward of the instrumental $\mathrm{O} \mathrm{K}$ edge.

We re-fitted the data, and obtained an improvement of $\Delta \chi^{2}=107$, for 20 additional degrees of freedom, with almost unchanged best-fitting parameters: $\Gamma=2.62 \pm 0.01$ and $N_{\mathrm{H}}=(3.60 \pm 0.05) \times 10^{20} \mathrm{~cm}^{-2}$. We measure a $(0.1-2) \mathrm{keV}$ flux of $F_{0.1-2}=(2.45 \pm 0.03) \times 10^{-11} \mathrm{erg} \mathrm{s}^{-1} \mathrm{~cm}^{-2}(1.2 \mathrm{mCrab})$. The LSF-smoothed residuals now appear flat over the entire 10-60 ̊ band (Figure 1, bottom panel).

\subsubsection{Unresolved Absorption Lines: Detectability of Carbon versus Oxygen}

A visual inspection of the LSF-smoothed residuals in Figure 1 (bottom panel) shows clear narrow (i.e., unresolved: $\Delta \lambda \lesssim 0.05 \AA$ ) deficit of counts involving 3-6 consecutive bins, especially in the spectral region where redshifted $\mathrm{C}$ absorption is expected. This is expected: indeed, the sensitivity of dispersed spectrometers with constant $\Delta \lambda$ and $\mathrm{S} / \mathrm{N}$ per resolution element, over a given bandpass, to a given ion column density, increases with wavelengths. This is because of the concurrence of two independent facts. First, the absorption-line equivalent width (EW) sensitivity of a dispersed spectrum with given constant $\Delta \lambda$ and $\mathrm{S} / \mathrm{N}$, down to a statistical significance $N \sigma$, can be written as follows:

$$
\mathrm{EW}_{\text {thresh }}=N_{\sigma}[\Delta \lambda /(\mathrm{S} / \mathrm{N})]
$$

Second, the absorption-line EW is related to the column density of the ion imprinting the line in the spectrum through the 
rest-frame position of the line and the redshift of the absorber. For unsaturated lines, we can write

$$
\mathrm{EW}\left(X^{i}\right) \simeq 8.9 \times 10^{-21} \times N_{X^{i}} \xi_{X^{i}} \lambda_{r f}^{2}(1+z),
$$

with EW in $\AA$ and where $N_{X^{i}}$ is the column density of the ion $i$ of the element $X$ in $\mathrm{cm}^{-2}, \xi_{X^{i}}$ is the oscillator strength of the given transition of the ion $X^{i}, \lambda_{\mathrm{rf}}$ is the rest-frame wavelength (in $\AA$ ) of the given transition of the ion $X^{i}$, and $z$ is the redshift of the absorber. Therefore, from Equations (1) and (2):

$$
N_{X^{i}}^{\text {Thresh }} \simeq 1.1 \times 10^{20} \times N_{\sigma} \xi_{X^{i}}^{-1} \lambda_{r f}^{-2}(1+z)^{-1} \Delta \lambda(\mathrm{S} / \mathrm{N})^{-1} .
$$

Let us now consider, the LETG spectrometer and, for example, the strongest $\mathrm{K} \alpha$ transitions of the two stable and most abundant ions of carbon and oxygen at $T \simeq 10^{6} \mathrm{~K}$, i.e., $\mathrm{C}$ V and $\mathrm{O}$ vII. The rest-frame wavelengths of these two transitions are in a 1:2 ratio, and fall in regions of the spectrometer with similar effective area (i.e., similar $\mathrm{S} / \mathrm{N}$ in the same spectrum). Therefore, from Equation (3), one gets

$$
\left[N_{\mathrm{C} \mathrm{V}}^{\text {Thresh }} / N_{\mathrm{O} \text { VII }}^{\text {Thresh }}\right] \simeq[\lambda(\mathrm{C} \mathrm{v} \mathrm{K} \alpha) / \lambda(\mathrm{O} \text { VII K } \alpha)]^{-2} \simeq 1 / 4 .
$$

For a broad range of WHIM temperatures, $\log T \simeq 5.5-6$, the fraction of $\mathrm{C} \mathrm{V}$ and $\mathrm{O}$ VII are similar and between 0.7 and 1 . At $5<\log T<5.5$, C v largely dominates over O viI. Moreover, in gas with solar-like composition, oxygen is roughly twice more abundant than carbon. So, for $5<\log T<6$, the minimum detectable $\mathrm{C}$ v column density is at least twice smaller than the minimum detectable $\mathrm{O}$ vII column density.

\subsubsection{Automated Identification of Absorption-line Candidates}

To identify candidate unresolved absorption lines we wrote a routine that scans the LSF-smoothed residuals (Figure 1, bottom panel) over $\Delta \lambda=125 \mathrm{~m} \AA$ wide contiguous bins $(2.5$ LETG resolution elements), to search for negative deficits of counts involving between 3 and 7 consecutive bins (i.e., 0.75-1.75 LETG LSF FWHM at our oversampled resolution), and line shaped, i.e., symmetrically distributed around a central negative peak (at least one left and one right bin with values higher than the peak). We do not consider resolved lines here (i.e., consecutive deficits of counts spreading over more than $\simeq 7$ bins), since we assume that any ISM or IGM absorption lines must have intrinsic FWHM $\ll 800 \mathrm{~km} \mathrm{~s}^{-1}$. By definition, LSF-smoothing of the residuals tends to smooth flickering in adjacent sub-resolution bins. Thus, on the one hand it highlights real absorption (or emission) lines, but on the other hand it also increases the number of false absorption (or emission) line-like features with typical LSF width (see, e.g., Figure 1, bottom panel). We can estimate the expected number of LSFshaped emission or absorption features exceeding a bin-by-bin integrated (in quadrature) significance of $3 \sigma$, by using a simple Gaussian argument. At our $4 \times$ LSF oversampled binning, we have a total of (60-10)/0.0125 = 4000 bins. Therefore, the Gaussian-predicted number of positive or negative LSFshaped fluctuations at $\geqslant 3 \sigma$ is $(1-0.997) \times 4000=12$. So, on average, we expect a total of six emission and six absorption LSF-shaped fluctuations. Indeed, the blind run of the scanning routine over the 10-60 ^ LSF-smoothed residuals (oversampled at a resolution of $0.25 \times$ the LETG LSF FWHM) finds exactly six LSF-shaped emission features exceeding the bin-by-bin integrated (in quadrature) statistical significance of $3 \sigma$, according to expectations given the blazar nature of the target and the consequent absence of intrinsic emission in the optical or infrared spectra of 1ES 1553+113. On the contrary, 50 of such features are found in absorption, clearly suggesting that real absorption lines are imprinted on the 10-60 A LETG continuum spectrum of $1 \mathrm{ES} 1553+113$. Given the way the scanning routine works, moving on contiguous 2.5 LETG-LSF wide bins, some of the 50 absorption-line candidates found by the routine are simply the same strong feature, detected twice over adjacent scanned bins. This leaves about 40 absorption-line candidates, still many more than predicted by Gaussian statistics and found in emission.

To confirm the real absorption-line nature of these candidates (at least down to the sensitivity of the current LETG spectrum), we therefore used unresolved (line FWHM frozen at $5 \mathrm{~m} \AA$ ) absorption Gaussians to model the non-smoothed data. We added these Gaussians one by one and refitted the data, by allowing the line position to vary within $0.5 \AA$ around the scanroutine determined position of the centroid. This procedure yielded the results summarized in Table 1 (Columns 1 and 2). Only 12 of the possible $\sim 40$ absorption candidates were actually confirmed by the fitting procedure at $>90 \%(1.6 \sigma)$ statistical significance. The single-line (prior to secure identification) statistical significances of 11 of these lines (computed as the ratio between the best-fitting line normalization and its $1 \sigma$ uncertainty) are in the range (2.2-4.1) $\sigma$ : three lines at $>4 \sigma$, three at $3.6 \leqslant \sigma<4$, and five at $2.2 \leqslant \sigma \leqslant 2.8$. (Table 1 , Column 2 and Figure 1, bottom panel). An additional line-like feature is only hinted by the data and can be fitted with a negative Gaussian with single-line statistical significance of $1.7 \sigma$. In the following, we consider the six lines with prior-to-identification statistical significance $\geqslant 3.6 \sigma(0.04$ and 0.08 expected by chance up to $z=0.4$ in the oxygen and carbon bands, respectively; see Section 4.2) as real absorption lines, which need to be identified. The remaining six absorption features are considered as tentative detections that need confirmation, and we consider them here only in association with either higher significance LETG absorbers or COS signposts, or both. In Figure 2, we show the best-fitting models of the 12 absorption lines superposed on the non-LSF-smoothed residuals (in standard deviations) of the data to the best-fitting continuum model. Best-fitting Gaussians models are color-grouped into six different systems, according to their possible identifications (Section 4.1).

\section{DISCUSSION}

The LETG spectrum of 1ES $1553+113$ reveals the presence of unresolved line-like absorption in both the $z<0.4$ oxygen $\mathrm{K} \alpha$ and carbon $\mathrm{K} \alpha$ regions. These absorption lines could be the imprints of either Galactic ISM and/or CGM, or of warmhot intervening IGM, or both. To identify these systems, we consider all the strongest ground-state metal transitions $(\mathrm{K} \alpha)$ from all ions of the two most abundant elements in gas with solar-like composition ( $\mathrm{O}$ and $\mathrm{C}$ ). We try to match the possible identifications, with the available redshift priors: (1) $z=0$, rest-frame absorption by the ISM of our Galaxy, or CGM (the LETG resolution does not allow us to kinematically distinguish between the two; e.g., Gupta et al. 2012, and references therein); and (2) the redshifts of the possible BLAs (or NLAs) and/or O VI absorbers identified in the COS spectrum of 1ES $1553+113$ (Danforth et al. 2010a).

\subsection{Absorption System Identifications}

The longest wavelength $\mathrm{H}_{\mathrm{I}} \mathrm{Ly} \alpha$ securely identified in the COS spectrum of $1 \mathrm{ES} 1553+113$, is at $\lambda \simeq 1695 \AA$ and $z=0.395$. At longer wavelengths, and up to the COS G160M 
Table 1

Absorption Lines in the LETG (and COS) Spectrum(a) of 1ES 1553+113

\begin{tabular}{|c|c|c|c|c|}
\hline $\begin{array}{l}\text { Wavelength } \\
\text { (§) }\end{array}$ & $\begin{array}{c}\text { Significance }{ }^{\mathrm{b}} \\
(\sigma)\end{array}$ & Redshift & Id & COS Hi, Metal I \\
\hline \multicolumn{5}{|c|}{ Galactic absorbers } \\
\hline $23.34 \pm 0.04$ & 2.5 & \pm 0.002 & O I molecular & $\mathrm{N} / \mathrm{A}$ \\
\hline $23.49 \pm 0.04$ & 4.1 & $-0.001 \pm 0.002$ & O I $\mathrm{K} \alpha$ atomic & $\mathrm{N} / \mathrm{A}$ \\
\hline $43.01 \pm 0.04$ & 2.8 & \pm 0.001 & $\mathrm{C}$ II $\mathrm{K} \alpha$ atomic & $\mathrm{N} / \mathrm{A}$ \\
\hline \multicolumn{5}{|c|}{ Intervening absorbers } \\
\hline \multicolumn{5}{|c|}{ High-confidence X-ray system detections and COS associations } \\
\hline $44.27 \pm 0.04$ & 4.1 & $0.312 \pm 0.001$ & $\mathrm{C}$ VI K $\alpha$ & BLA-O VI $z_{\mathrm{FUV}}=0.3113$ \\
\hline $52.82 \pm 0.04$ & 4.1 & $0.312 \pm 0.001$ & $\mathrm{C} v \mathrm{~K} \alpha$ & BLA-O VI $z_{\mathrm{FUV}}=0.3113$ \\
\hline $49.80 \pm 0.04$ & 3.9 & $0.237 \pm 0.001$ & $\mathrm{C} v \mathrm{~K} \alpha$ & $\mathrm{BLA} z_{\mathrm{FUV}}=0.23666,0.23734$ \\
\hline$[26.65 \pm 0.04$ & 1.7 & $0.234 \pm 0.002$ & $\mathrm{O}$ VII K $\alpha$ & BLA $\left.z_{\mathrm{FUV}}=0.23666,0.23734\right]^{\mathrm{c}}$ \\
\hline $45.58 \pm 0.04$ & 3.8 & $0.132 \pm 0.001$ & $\mathrm{C} v \mathrm{~K} \alpha$ & $\mathrm{BLA}^{\mathrm{d}} z_{\mathrm{FUV}}=0.1333$ \\
\hline $38.25 \pm 0.04$ & 2.7 & $0.134 \pm 0.001$ & $\mathrm{C}$ vi K $\alpha$ & $\mathrm{BLA}^{\mathrm{d}} z_{\mathrm{FUV}}=0.1333$ \\
\hline \multicolumn{5}{|c|}{ Tentative X-ray detections and COS associations } \\
\hline $47.68 \pm 0.04$ & 3.6 & $0.184 \pm 0.001$ & $\mathrm{C} \vee \mathrm{K} \alpha$ & Triple-H I: $z$ FUV $=0.186-190$ \\
\hline $47.94 \pm 0.04$ & 2.2 & $0.191 \pm 0.001$ & $\mathrm{C} \vee \mathrm{K} \alpha$ & BLA-O VI $z_{\mathrm{FUV}}=0.18989$ \\
\hline$[26.65 \pm 0.04$ & 1.7 & $0.191 \pm 0.002$ & $\mathrm{O} \vee \mathrm{K} \alpha$ & BLA-O VI $\left.z_{\mathrm{FUV}}=0.18989\right]^{\mathrm{c}}$ \\
\hline $22.48 \pm 0.04$ & 2.3 & $0.041 \pm 0.002$ & $\mathrm{O}$ vII $\mathrm{K} \alpha$ & $\mathrm{BLA} z_{\mathrm{FUV}}=0.0428$ \\
\hline
\end{tabular}

Notes.

a We use $40 \mathrm{mÅ}$ as $1 \sigma$ systematic uncertainties for the HRC-LETG co-added negative- and positive-order wavelength scale. This is (4/5) of the LETG line-spread function FWHM, and it is due to the nonlinearity in the HRC-LETG effective dispersion relation.

${ }^{\mathrm{b}}$ Single-line statistical significances, obtained by ratioing the best-fitting line normalization and its $1 \sigma$ error.

${ }^{\mathrm{c}}$ This line can be identified with either an $\mathrm{O}$ VII $\mathrm{K} \alpha$ transition at $z_{X}=0.234 \pm 0.002$, close but only $3 \sigma$ consistent with the $\mathrm{C}$ v-BLA system at $z_{X}=0.237 \pm 0.001$ (Section 4.1.3), or with an O v K $\alpha$ transition associated with the C v-BLA-O vi system at $z_{X}=0.191 \pm 0.001$ (Section 4.1.5).

${ }^{\mathrm{d}}$ Given the relatively low-temperature derived from the $\mathrm{H}$ I Doppler parameter, the column density of this $\mathrm{H}$ I component is probably too low to produce all the $\mathrm{C} v$ and $\mathrm{C}$ VI detected in the LETG, even assuming solar abundances (see Section 4.1.3).

band-limit $(\lambda \sim 1800 \AA$, or $z \sim 0.48$ in $\mathrm{H}$ I Ly $\alpha)$, the absorptionline density drops dramatically, indicating $z \simeq 0.4$ as a likely redshift for the target (Danforth et al. 2010a). We therefore assume $z=0.4$ as the redshift of 1ES $1553+113$.

The 12 absorption features identified as possible lines in the LETG spectrum of 1ES 1553+113, have wavelengths between $\lambda=22.48-26.65 \AA$ (four lines in the O-region of the spectrum) and $\lambda=38.25-52.82 \AA$ (eight lines in the C-region of the spectrum). The shortest wavelength $\mathrm{K} \alpha$ transition of $\mathrm{O}$ is that of its H-like ion (O VIII), at $\lambda=18.97 \AA$ (Verner et al. 1996), while the longest wavelength $\mathrm{K} \alpha$ transition of $\mathrm{O}$ is the inner-shell transition of its neutral ion (O I), at $\lambda=23.52 \AA$ (E. Behar 2006, 2011, private communication). ${ }^{23}$ Similarly, for $\mathrm{C}$, we have $\lambda\left(\mathrm{C}_{\left.\mathrm{VI}_{\mathrm{L} \alpha}\right)}\right)=33.74 \AA$ (Verner et al. 1996), and $\lambda\left(\mathrm{C}_{\mathrm{I}_{\alpha} \alpha}\right)=43.37$ and $43.27 \AA$ (an LETG-resolved doublet; E. Behar 2006, 2011, private communication), as the shortest and longest wavelength transitions, respectively. Intermediate ionization ions have their $\mathrm{K} \alpha$ transition wavelengths between these extremes. So, the four LSF-shaped features in the 22-27 range (Figure 1, top panel), if true absorption lines, can only be identified with $\mathrm{O}$ VIII in the ranges $0.185<z<0.4$ or with

\footnotetext{
23 For most of the $\mathrm{K} \alpha$ inner-shell transitions of $\mathrm{C}$ and $\mathrm{O}$ (with the exception of $\mathrm{O}$ I and $\mathrm{C}$ II, for which empirical observational values are available, e.g., Takei et al. 2002; Schulz et al. 2003; Juett et al. 2004), we use wavelengths and oscillator strengths computed through the HULLAC code (Bar-Shalom et al. 2001). The uncertainties on the rest-frame positions of these transitions are of the order of $0.1 \%-1 \%$ (i.e., $\Delta \lambda \sim 20-200 \mathrm{m \AA}$; Behar \& Netzer 2002). However, several of these positions have been benchmarked with either laboratory or astrophysical experiments (e.g., Kaastra et al. 2011, and reference therein; Schulz et al. 2003, and references therein; Juett et al. 2004) and have resulted to be accurate to $\Delta \lambda \sim 2-10 \mathrm{~m} \AA$.
}

O I-O VII at $0 \lesssim z<0.4$, with the exact lower limit depending on the ion considered. Analogously, the eight line-like features at $\lambda \geqslant 38.25 \AA$ can only be identified with $\mathrm{C}$ VI in the range $0.134<z<0.4$ or $\mathrm{C}$ I- $\mathrm{C}$ v at $0 \lesssim z<0.4$, while the feature at $38.25 \AA$ can only be due to redshifted C VI.

We have checked these ions one by one, and propose possible identifications based on $\mathrm{H}$ I and/or O VI COS associations. We only check here for possible X-ray $\mathrm{C}$ and/or $\mathrm{O}$ counterparts to the FUV signposts. These are by far the two most abundant metals in gas with solar-like composition (Asplund et al. 2009), with $\mathrm{C}$ being only a factor of $\sim 2$ less abundant than $\mathrm{O}$, and the third most abundant metals $(\mathrm{N}$ and $\mathrm{Ne}$ ) being $\sim 4 \times$ less abundant than C. Similarly, when in doubt between two or more identifications involving different ions of $\mathrm{C}$ and/or $\mathrm{O}$, we always favor the He-like metal identification, i.e., $\mathrm{C} \mathrm{v}$ and/or O vII. These are the He-like ions of $\mathrm{C}$ and $\mathrm{O}$, respectively, and as such are highly stable and by far the most abundant ions of their respective elements over a broad range of temperatures. The oscillator strengths of their $\mathrm{K} \alpha$ transitions are also the strongest of all the other $\mathrm{K} \alpha$ transitions from lower- or higher-ionization ions.

\subsection{1. $z \simeq 0$ Galactic Absorption}

The three lines at $\lambda=23.34 \AA, \lambda=23.49 \AA$, and $\lambda=$ $43.01 \AA$ are consistent with $z \simeq 0$ absorption by neutral (or only moderately ionized) species of $\mathrm{C}$ and $\mathrm{O}$. In particular, we clearly $(4.1 \sigma)$ detect atomic O I at $\lambda=23.49 \pm 0.04 \AA$ and also a marginal $(2.5 \sigma)$ excess of molecular O I (possibly ISM oxygen in compound forms of oxide dust grains; e.g., Takei et al. 2002) at $\lambda=23.34 \pm 0.04 \AA$, with respect to the instrumental 

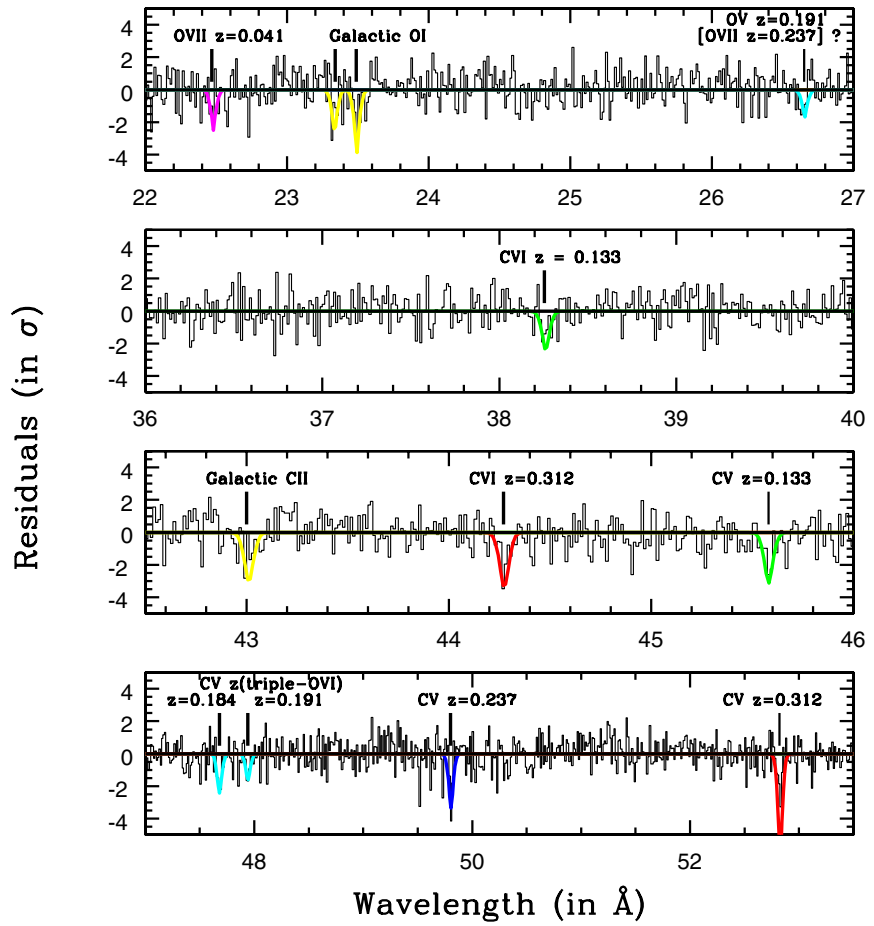

Figure 2. Non-LSF-smoothed residuals (in $\sigma$ ) of four portions of the $500 \mathrm{ks}$ LETG spectrum of 1ES 1553+113, where Galactic O I and C II, as well as intervening $\mathrm{O}$ VII and $\mathrm{CV}$, are tentatively detected, at single-line statistical significances in the $2.2 \sigma-4.1 \sigma$ range. Best-fitting curves of different colors identify different systems: (1) Galactic O I and C II (yellow), (2) O VII at $z_{X}=0.041$ (magenta), (3) $\mathrm{CV}$ (and maybe O viI) at $z_{X}=0.237$ (blue), (4) $\mathrm{C}$ VI and $\mathrm{C}$ V at $z_{X}=0.312$ (red), (5) $\mathrm{C} \mathrm{V}$ and $\mathrm{C} \mathrm{VI}$ at $\left\langle z_{X}\right\rangle=0.133$ (green), and (6) $\mathrm{CV}$ at the COS triple-H I-metal redshifts (cyan).

molecular O I. Atomic O I is not an instrumental feature (e.g., Takei et al. 2002). Finally, we marginally (2.8\%) detect the signature of $\mathrm{C}_{\mathrm{II}}$ (whose $\mathrm{K} \alpha$ transition is twice as strong as the single transitions of the resolved and undetected $\mathrm{C}_{\mathrm{I}}$ doublet) at $\lambda=43.01 \pm 0.04 \AA{ }^{24}$ We identify these three transitions as due to the cold ISM of our Galaxy (Table 1, Columns 3 and 4 and Figures 1 and 2, yellow lines), and we therefore consider their single-line statistical significance as their true (i.e., after a secure identifications) statistical significance.

We also note that we do not detect any $\mathrm{O}_{\text {VII }} \mathrm{K} \alpha z \simeq 0$ absorber along the line of sight to $1 \mathrm{ES} 1553+113$, down to the $3 \sigma$ sensitivity limit of our Chandra-LETG spectrum, in this spectral region: $\mathrm{EW}(\mathrm{O} \mathrm{VII}(z=0))<12 \mathrm{m \AA}$.

Below we present the possible intervening system identifications in order of statistical significances.

\subsubsection{The $z_{X}=0.312 \pm 0.001 C$ V-C VI-BLA-O vI WHIM System}

The line at $\lambda=44.27 \AA$ is that detected at the highest statistical significance $(4.1 \sigma)$ in the LETG spectrum (together with the line at $\lambda=52.82 \AA$ and the Galactic O I atomic line). The $\mathrm{C}_{\mathrm{I}}, \mathrm{C}_{\mathrm{II}}$, and $\mathrm{C}_{\mathrm{IV}} \mathrm{K} \alpha$ transitions, at this wavelength, would be redshifted at $z_{X}=0.021,0.031$, and 0.070 . At none of these redshifts is there a metal or $\mathrm{H}_{\mathrm{I}}$ association in COS. At the redshift of a putative $\mathrm{C}$ III, instead, $z_{X}=0.051$, there is a moderately strong NLA. However, no imprint of a C III $\lambda 977$ absorber is seen in the FUSE spectrum of 1ES 1553+113 (though

\footnotetext{
24 Not only the $\mathrm{C}_{\text {II }} \mathrm{K} \alpha$ transitions is intrinsically stronger than the $\mathrm{C}_{\mathrm{I}} \mathrm{K} \alpha$, but also $\mathrm{C}_{\mathrm{II}}$ is expected to be much more abundant than $\mathrm{C}_{\mathrm{I}}$ in the Galaxy, because neutral carbon is easily photoionized by ambient $\mathrm{UV}$ radiation (the $\mathrm{C}_{\mathrm{I}}$ ionization edge is at $11.260 \mathrm{eV}$ or $1101 \AA$ ).
}

its presence cannot be ruled out due to the presence of $\mathrm{Hi} \mathrm{Ly} \beta$ and $\mathrm{O}$ I absorption/airglow in FUSE), and no other metal lines are present in the FUSE or COS spectra at this redshift. $\mathrm{C} \mathrm{V}$ and $\mathrm{C}$ VI identifications are more promising.

The $\mathrm{C}$ v K $\alpha$ transition would be redshifted at $z_{X}=0.100 \pm$ 0.001 . At a close (but $>3 \sigma$ inconsistent) redshift $\left(z_{\mathrm{FUV}}=\right.$ 0.10230 ) there is one of the strongest $\mathrm{HI}$ Ly $\alpha$ lines in COS, which is also seen in $\operatorname{Ly} \beta$ and is consistent with being thermally broadened (BLA) at $T \simeq 10^{5} \mathrm{~K}$. Moreover, at $\lambda=45.58 \AA$ there is another line in the LETG spectrum $(3.8 \sigma)$, which, if identified with $C_{\text {IV }}$, would fall at $z_{X}=0.100 \pm 0.001$. The possible temperature of the $\mathrm{H}$ I absorber at $z_{\mathrm{FUV}}=0.10230$ is consistent with the presence of $\mathrm{CIV}$ and $\mathrm{C} \mathrm{V}$, however no C IV doublet ( $\lambda=1548.195$ and 1550.77$)$ is seen in the COS spectrum at $z=0.10230$ or $z=0.100$. We conclude (both for the lack of C IV in COS and the redshift inconsistency with the BLA) that the $z_{X}=0.100 \mathrm{Cv} / \mathrm{C}$ IV identification with a thick and moderately cool WHIM filament traced by the COS BLA at $z_{\mathrm{FUV}}=0.10230$, is unlikely.

A more likely identification is with a $\mathrm{C}$ vI $\mathrm{K} \alpha$ transition at $\lambda=44.27 \AA$, which would have a redshift of $z_{X}=0.312$. At $\lambda=52.82 \AA$, there is the other highest statistical significance line of the LETG spectrum $(4.1 \sigma)$, which, if identified with $\mathrm{C} v \mathrm{~K} \alpha$, would fall at $z_{X}=0.312 \pm 0.001$, exactly the redshift of the $\mathrm{C}$ vi identification. No H I or $\mathrm{O}$ vi identification is reported at a consistent redshift in the published COS spectrum. However, with additional data, the currently available COS spectrum of 1ES $1553+113$ has an $\mathrm{S} / \mathrm{N}$ of a factor $\sim 1.5-2 \times$ higher than the published spectrum. We therefore downloaded from the HST-MAST archive all available HST-COS spectra of 1ES $1553+113$ taken with the G130M and G160M gratings, and re-analyzed the two portions of the spectrum at $1350-1365 \AA$ and 1590-1600 $\AA$, regions where the $\mathrm{OVI}\left(1 s^{2} 2 s \rightarrow 1 s^{2} 2 p\right)$ doublet (i.e., $\mathrm{O} \mathrm{VI}_{1}(\lambda 1031.9)$, oscillator strength $f=0.133$, and $\mathrm{O} \mathrm{VI}_{2}(\lambda 1037.6), f=0.066$ ) and the $\mathrm{H} \mathrm{I} \mathrm{Ly} \alpha$ at $z \sim 0.308-0.316$ are supposed to lie.

We identify a strong $\mathrm{H}_{\mathrm{I}} \mathrm{BLA}$ at $z_{\mathrm{FUV}}=0.31124 \pm 0.00003$ (8.1 $\sigma$; Figure 3, top panel), with the following best-fitting parameters: $\lambda=1594.03 \pm 0.03 \AA, b=60 \pm 6 \mathrm{~km} \mathrm{~s}^{-1}$, and $\mathrm{EW}=138 \pm 17 \mathrm{m \AA}$. The strongest line of the $\mathrm{O}$ v doublet $\left(\mathrm{O} \mathrm{VI}_{1}\right)$ is also detected $(3.9 \sigma$; Figure 3 , central panel), with best-fitting parameters: $\lambda=1353.13 \pm 0.04 \AA$ (i.e., $\left.z_{\mathrm{OVI}}=0.31130 \pm 0.0004\right), b=32 \pm 7 \mathrm{~km} \mathrm{~s}^{-1}$, and $\mathrm{EW}=$ $27.5 \pm 7.0 \mathrm{~mA}$. Finally, the weakest line of the $\mathrm{O}$ VI doublet $\left(\mathrm{O} \mathrm{VI}_{2}\right)$ is not detected in the data (Figure 3, bottom panel), but the $\operatorname{COS} 3 \sigma \mathrm{EW}$ upper limit at the wavelengths of this line, $\mathrm{EW}<21 \mathrm{m \AA}$, is consistent with the maximum possible EW expected for the $\mathrm{OVI}_{2}$ (given the measured $\mathrm{EW}\left(\mathrm{O} \mathrm{VI}_{1}\right)$ and the fact that this line is not blank). From the observed $\mathrm{H}$ I and $\mathrm{O}$ VI Doppler parameters, we can estimate the relative contribution of thermal and turbulence broadening. In general, if $b_{\mathrm{HI}}^{\mathrm{Obs}}$ and $b_{X}^{\mathrm{Obs}}$ are the observed Doppler parameters of two associated lines of H I and a metal $X$ with atomic weight $A$, then the Hi thermal parameter is given by

$$
\left(b_{\mathrm{H} \mathrm{I}}^{\mathrm{therm}}\right)^{2}=[A /(A-1)] \times\left[\left(b_{\mathrm{H} \mathrm{I}}^{\mathrm{Obs}}\right)^{2}-\left(b_{X}^{\mathrm{Obs}}\right)^{2}\right] .
$$

So, in this case we have $b_{\mathrm{H}_{\mathrm{I}}}^{\text {therm }}=52 \pm 7 \mathrm{~km} \mathrm{~s}^{-1}$ (implying $\log T=5.2 \pm 0.1)$ and $b_{\text {turb }}=30 \pm 14 \mathrm{~km} \mathrm{~s}^{-1}$, with a thermal contribution (in quadrature) of $75 \%$ and a $b_{\mathrm{HI}}^{\text {Obs }} / b_{\mathrm{HI}}^{\text {therm }}=1.15$ correction factor, similar to the average $b_{\mathrm{HI}}^{\mathrm{Obs}} / b_{\mathrm{HI}}^{\text {therm }}=1.2$ value found by Danforth et al. (2010b).

We conclude that the two highest significance intervening LETG lines are $\mathrm{C} \mathrm{V}$ and $\mathrm{C}$ VI absorbers at $z_{X}=0.312$ (Table 1; 


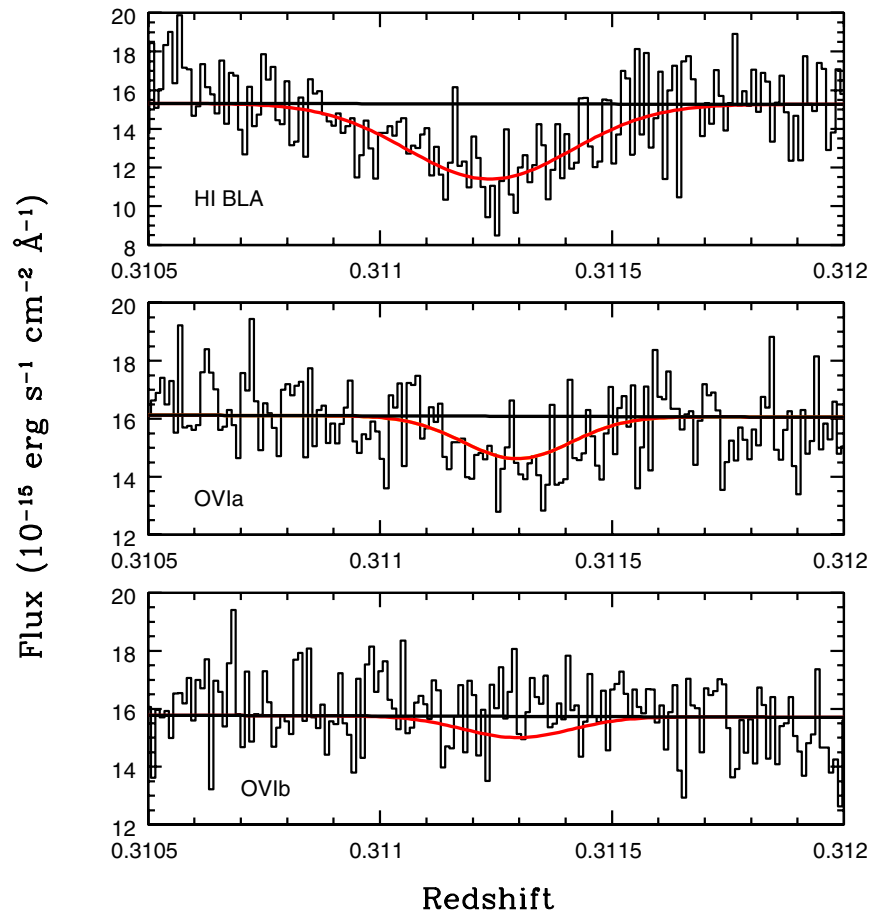

Figure 3. Three portions of the COS spectrum of $1 \mathrm{ES} 1553+113$, centered around the $\mathrm{HI}_{\mathrm{I}} \mathrm{Ly} \alpha$ (top panel), $\mathrm{O} \mathrm{VI}_{1}$ (central panel), and $\mathrm{O} \mathrm{VI}_{2}$ (bottom panel), at $z_{\mathrm{FUV}}=0.31124 \pm 0.00003$. In all panels, red curves are the best-fitting Gaussians for the $\mathrm{HI}_{\mathrm{I}} \mathrm{Ly} \alpha$ (top panel), $\mathrm{OVI}_{1}$ (central panel), and $\mathrm{OVI}_{2}$ (bottom panel). $\operatorname{COS}$ data are binned at about $1 / 8$ the $\operatorname{COS}$ resolution, i.e., $\Delta \lambda \simeq 9 \mathrm{~m} \AA$. (A color version of this figure is available in the online journal.)

Figure 1, red lines), possibly imprinted by an intervening WHIM filament, as further suggested by the a posteriori discovery of an associated strong BLA and O VI counterpart in the COS spectrum, with the right temperature (i.e., line Doppler parameters) and strength. This identification is further strengthened by the a posteriori discovery of two additional associated X-ray absorption lines. Figure 4 shows five portions of the LETG spectrum of $1 \mathrm{ES} 1553+113$, centered around the $\mathrm{CV} \mathrm{K} \alpha$ (top panel), $\mathrm{C}$ vI $\mathrm{K} \alpha$ (second panel), $\mathrm{Cv} \mathrm{K} \beta$ (third panel), Ov K $\alpha$, and $\mathrm{O}$ vII $\mathrm{K} \alpha$ transitions at $z=0.312$. Clearly, both the $\mathrm{O} v \mathrm{~K} \alpha$ and the $\mathrm{C} v \mathrm{~K} \beta$ lines are hinted in the data, and were not picked up by our scanning routine because of their bin-by-bin integrated (in quadrature) significance, which is slightly lower $(2.7 \sigma$ for both lines) than the $3 \sigma$ threshold that we imposed in our search (Section 3.2). The single-line statistical significance of these two additional lines are $1.9 \sigma$ and $1.6 \sigma$, respectively. The $\mathrm{O}$ VII $\mathrm{K} \alpha$ transition, instead, is undetected (Figure 4, bottom panel) down to the $3 \sigma$ sensitivity threshold of $\mathrm{EW}_{\mathrm{O}_{\mathrm{VII}}}(z=0.312)=20 \mathrm{~m} \AA$, confirming the relatively low temperature for this system.

$$
\text { 4.1.3. The } z_{X}=0.237 \pm 0.001 \text { WHIM System }
$$

The line at $\lambda=49.80 \AA$ is the second highest statistical significance line detected with the LETG $(3.9 \sigma)$. This line can only be associated with a COS absorber if identified as a $\mathrm{C} \mathrm{v} \mathrm{K} \alpha$ at $z_{X}=0.237 \pm 0.001$. Indeed, at a marginally consistent redshift of $z_{\mathrm{FUV}}=0.23559$, there is a weak $(\mathrm{EW}=20 \pm 7 \mathrm{m \AA})$ NLA $\left(b=12 \pm 8 \mathrm{~km} \mathrm{~s}^{-1}\right)$ in the published COS spectrum (Danforth et al. 2010a). The Hi line, however, is too weak and much too narrow to be considered the actual counterpart of a putative $\mathrm{CV}$ absorber, so, if the LETG identification is correct, $\mathrm{CV}$ and NLA would be the tracers of a spatially co-located multi-phase WHIM filament, and a broader $\mathrm{HI}_{\mathrm{I}}$

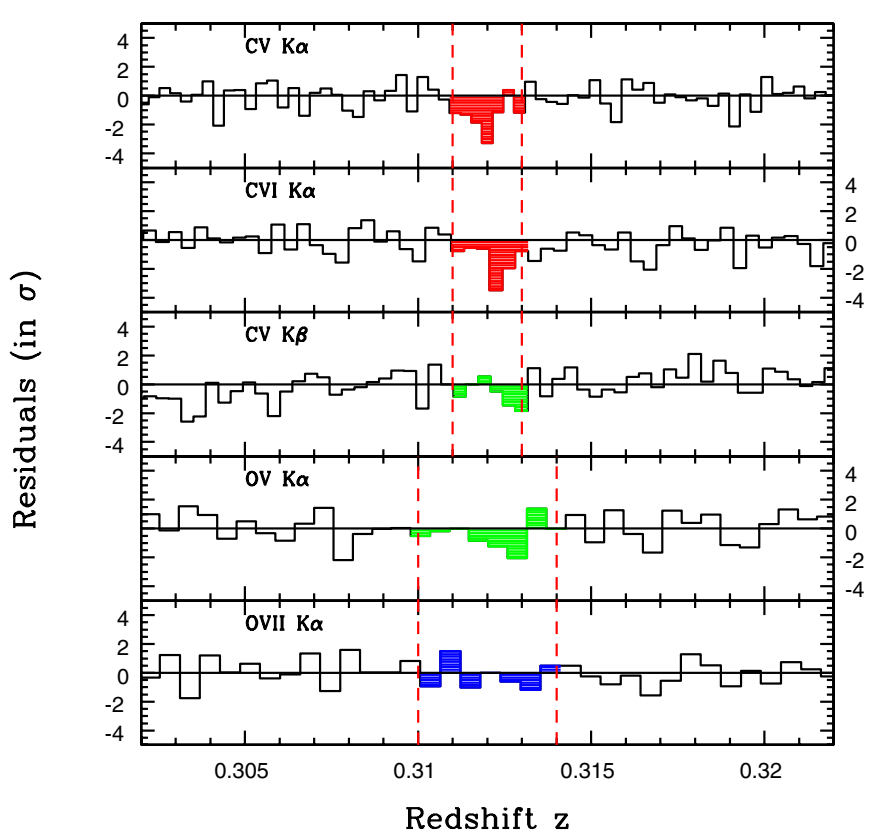

Figure 4. Five portions of the LETG spectrum of 1ES 1553+113, centered around the X-ray redshift of the $\mathrm{C}$ V-C VI system, for $\mathrm{Cv} \mathrm{K \alpha}$ (top panel), $\mathrm{C}$ VI K $\alpha$ (second panel), $\mathrm{C} v \mathrm{~K} \beta$ (third panel), O v K $\alpha$, and O vII K $\alpha$. Detected lines are filled in red, lines hinted in the data are filled in green, while nondetections have the spectral bins filled in blue. The two vertical dashed red lines delimit the X-ray redshift of the system: the wider interval for oxygen lines, compared with carbon lines, is due to the $\Delta \lambda=40 \mathrm{~m} \AA$ systematic uncertainty we assume throughout the entire LETG band, which translates into $\Delta z=0.001$ and $\Delta z=0.002$ at the wavelengths of the carbon and oxygen transitions, respectively.

(A color version of this figure is available in the online journal.)

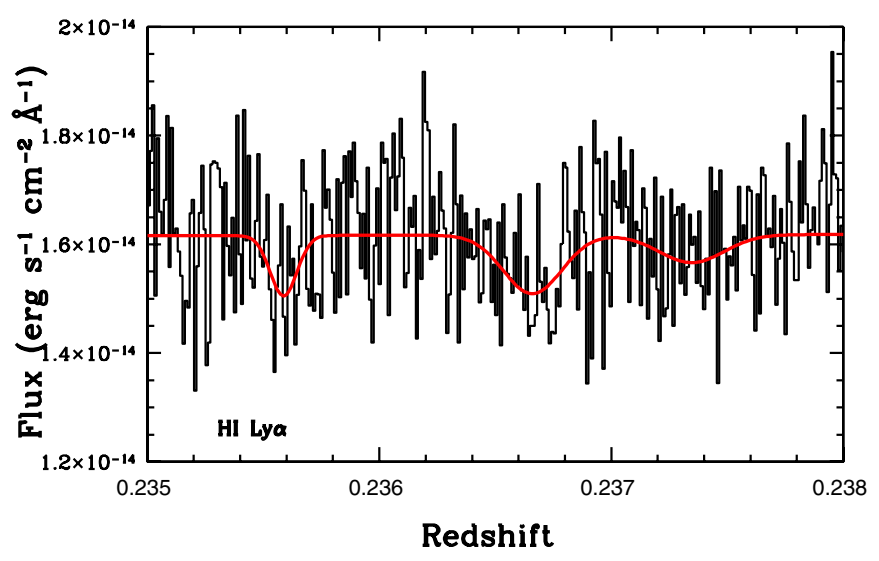

Figure 5. Portion of the COS spectrum of 1 ES $1553+113$ where the $\mathrm{HI}_{\mathrm{I}}$ Ly $\alpha$ transition at $z_{\mathrm{FUV}}=0.235-0.238$ lies. The red curve is the bestfitting continuum model plus three negative Gaussians modeling: (a) an NLA at $z_{\mathrm{FUV}}=0.23559$, (b) a BLA at $z_{\mathrm{FUV}}^{1}=0.23666$, and (c) a BLA at $z_{\mathrm{FUV}}^{2}=0.23734$.

(A color version of this figure is available in the online journal.)

absorber should exist at $z \sim 0.237$, undetected down to the sensitivity limit of the published COS spectrum. To investigate this possibility further, we searched for such a BLA absorber in the currently available COS spectrum of 1ES $1553+113$, which, at the interesting wavelengths has a factor of two higher $\mathrm{S} / \mathrm{N}$ than the published spectrum. We confirm the presence of the NLA at $z_{\mathrm{FUV}}=0.23559$ with $\mathrm{EW}=12 \pm 4 \mathrm{m \AA}$ and $b=34_{-10}^{+17} \mathrm{~km} \mathrm{~s}^{-1}$ (Figure 5). Additionally, the data also show a structured and broad absorption complex at $z \simeq 0.2362-0.2378$ (Figure 5). We model this complex with two Gaussians with 


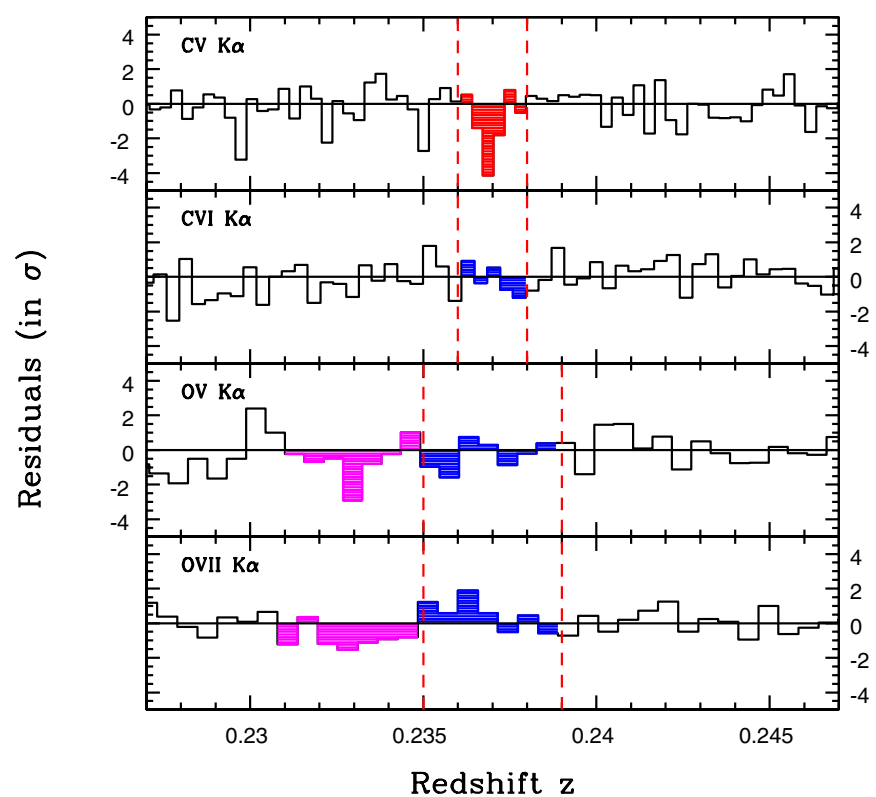

Figure 6. Four portions of the LETG spectrum of 1ES 1553+113, centered around the X-ray redshift of the $\mathrm{Cv} \mathrm{K} \alpha z=0.237 \pm 0.001$ identification, for $\mathrm{C}$ v K $\alpha$ (top panel), $\mathrm{C}$ vi K $\alpha$ (second panel), O v K $\alpha$ (third panel), and O vII K $\alpha$ (bottom panel). Detected lines are filled in red. Non-detections have the spectral bins filled in blue. The two line-like features in the third and bottom panels, filled in magenta, are possible $\mathrm{O} \vee \mathrm{K} \alpha$ and $\mathrm{O}$ vII $\mathrm{K} \alpha$ identifications at a redshift close to (but only marginally consistent with) that of the $\mathrm{C} v$-BLA system. The two vertical dashed red lines delimit the X-ray redshift interval of the $\mathrm{C}$ V-BLA system.

(A color version of this figure is available in the online journal.)

best-fitting $\mathrm{H}_{\mathrm{I}} \mathrm{Ly} \alpha$ centroids $z_{\mathrm{FUV}}^{1}=0.23666, z_{\mathrm{FUV}}^{2}=0.23734$, equivalent widths $\mathrm{EW}_{1}=25 \pm 5 \mathrm{~m} \AA(5 \sigma), \mathrm{EW}_{2}=13 \pm 6 \mathrm{~m} \AA$ $(2.2 \sigma)$, and Doppler parameters $b_{1}=72 \pm 20 \mathrm{~km} \mathrm{~s}^{-1}$ and $b_{2}=80_{-20}^{+40} \mathrm{~km} \mathrm{~s}^{-1}$. The redshifts of these two BLAs are both fully consistent with that of the putative $\mathrm{Cv} \mathrm{K} \alpha$ absorber, and their widths suggest temperatures in the ranges $\log T_{1}=5.1-5.7$ and $\log T_{2}=5.2-5.9$ (where the lower boundaries of these intervals are corrected for the average observed $b / b_{\text {therm }}=1.2$ ratio: Danforth et al. 2010a), again consistent with the presence of a large fraction $(\sim 60 \%-80 \%)$ of $\mathrm{C} \mathrm{V}$.

In the same temperature interval $\mathrm{CVI}$ is also relatively abundant, with a fraction raising monotonically from $f_{\mathrm{CVI}} \simeq$ $15 \%$ at $\log T=5.1$ to $f_{\mathrm{CVI}} \simeq 50 \%$ at $\log T=5.9$, but the oscillator strength of the $\mathrm{C}$ VI $\mathrm{K} \alpha$ transition is 1.7 times smaller than that of the $\mathrm{CvK} \alpha$ transition. The strongest transitions expected from oxygen, instead, depend on the exact temperature within this broad interval: at $\log T \simeq 5.1-5.4, \mathrm{OV} \mathrm{K} \alpha$ is the strongest transition expected (with $f_{\mathrm{Ov}} \simeq 10 \%-40 \%$ ), while at $\log T=\simeq 5.5-5.9$ the $\mathrm{O}$ vII $\mathrm{K} \alpha$ dominates, with $f_{\text {OVII }} \simeq 70 \%-90 \%$. We therefore looked for the presence of these three transitions in the LETG spectrum of 1ES 1553+113. The top panel of Figure 6 shows the detection that we tentatively identify with $\mathrm{C} v \mathrm{~K} \alpha$ at $z_{X}=0.237$. The remaining three panels show the positions of the $\mathrm{C}$ vI K $\alpha$ (second panel), O v K $\alpha$ (third panel), and $\mathrm{O}$ vII $\mathrm{K} \alpha$ (bottom panel) in the $z_{X}=0.227-0.247$ redshift range. We note that $\mathrm{Ov} \mathrm{K} \alpha$ and $\mathrm{C} v \mathrm{KI} \alpha$ (shifted by $\Delta z \simeq+0.001$ ) may be hinted at in the data (second and third panels of Figure 6). We also note that the two line-like deficits of counts visible in the third and bottom panels of Figure 6 (magenta bins), just leftward of the $90 \%$ confidence redshift interval of our tentative $\mathrm{C} v \mathrm{~K} \alpha$ identification (dashed red lines), could be identifiable with $\mathrm{Ov} \mathrm{K} \alpha$ and $\mathrm{O}$ vII $\mathrm{K} \alpha$ at $z_{X}=0.233 \pm 0.002$, respectively. Indeed the magentacolored feature in the bottom panel of Figure 6 is the lowest significance $(1.7 \sigma)$ line-like absorption feature identified by our scanning routine and subsequently confirmed by a Gaussian fit in the LETG, at $\lambda=26.65 \AA$. However, the redshift of these two putative $\mathrm{O} v \mathrm{~K} \alpha$ and $\mathrm{O}$ VII $\mathrm{K} \alpha$ identifications is only marginally $(3 \sigma)$ consistent with that of our $\mathrm{C} \mathrm{v} \mathrm{K \alpha}$ identification. Moreover, for the putative $\mathrm{O}$ VII $\mathrm{K} \alpha$ at $z=0.233 \pm 0.002$ an alternative identification is possible: that of an $\mathrm{O} v \mathrm{~K} \alpha$ absorber at $z=0.191 \pm 0.002$ associated with a C V-BLA system (see Section 4.1.5). Higher quality data are needed to discriminate between these two possibilities.

We conclude that the most likely identification for the line at $\lambda=49.80 \AA(3.9 \sigma)$ is that of a $\mathrm{C} \mathrm{V} \mathrm{K} \alpha$ line imprinted by a highly ionized shock-heated WHIM metal system at $z_{X}=0.237 \pm 0.001$, producing also structured BLA absorption at $z_{\mathrm{FUV}}^{1}=0.23666$ and $z_{\mathrm{FUV}}^{2}=0.23734$ (Figure 5), as well as a nearby NLA at $z_{\mathrm{FUV}}=0.23559$ (Figure 5) in a mildly photoionized portion of the filament, possibly at the interface of the shock-heated part of the main-body filament (Table 1; Figure 1, blue lines; Figures 5 and 6). Such multi-phase systems, with metals tracing hot collisionally ionized gas and narrow $\mathrm{H}$ I tracing a cooler photoionized portion of the same system, have been observed in O VI-NLA in the FUV (Danforth \& Shull 2008), but it would be the first time they are observed in hotter gas traced by $\mathrm{C} v-\mathrm{BLA}$ pairs. Confirming the presence of a nearby higher-ionization $\mathrm{O}$ v-O vII absorber, would probe an even more extreme regime and would therefore be extremely important.

\subsubsection{The Multi-phase and Multi-component $\left\langle z_{X}\right\rangle=0.133 \pm 0.002$} $C$ V-C VI-BLA System

The line at $\lambda=45.58 \AA(3.8 \sigma)$ cannot be associated with any metal or $\mathrm{H}_{\mathrm{I}}$ system in COS, if identified as $\mathrm{C}_{\mathrm{II}}, \mathrm{C}$ III, or $\mathrm{C}$ VI. The $\mathrm{C}$ IV identification has already been ruled out in Section 4.1.2, because no corresponding C IV transition is detected in $\operatorname{COS}$ at $z=0.100 \pm 0.002$. At the redshift of a putative $C_{\text {I }}$ identification $\left(z_{X}=0.051\right)$, instead, there is an NLA in $\operatorname{COS}\left(z_{\mathrm{FUV}}=0.05094\right)$. However, $\mathrm{C}$ I must be associated with almost neutral gas, with a large $\mathrm{HI} / \mathrm{H}$ fraction. The $\mathrm{H}$ i column derived from the COS data is only $N_{\mathrm{HI}}=2 \times 10^{13} \mathrm{~cm}^{-2}$, implying a total baryon column of at most $N_{\mathrm{H}} \sim 10^{15} \mathrm{~cm}^{-2}$. Galactic $\mathrm{C}_{\mathrm{I}}$ is not detected in these LETG data, and our line of sight to $1 \mathrm{ES} 1553+113$ crosses a Galactic column density $N_{\mathrm{H}}=3.7 \times 10^{20} \mathrm{~cm}^{-2}$. Finally, there are $10 \mathrm{C}_{\mathrm{I}}$ transitions accessible in the COS bandpass, and none is detected at $z=0.051$. We therefore rule out the $\mathrm{C}_{\mathrm{I}}$ identification for this line.

The only possibility left is $\mathrm{C} v$ at $z_{X}=0.132 \pm 0.001$. The marginal detection at $\lambda=38.25 \AA(2.7 \sigma)$ would have a marginally consistent redshift of $z_{X}=0.134 \pm 0.001$, if identified with $\mathrm{C}$ VI $\mathrm{K} \alpha$. At a redshift consistent with both the $\mathrm{CV}$ and $\mathrm{CVI} \mathrm{X}$-ray lines $\left(z_{\mathrm{FUV}}=0.13324\right)$, there is a weak $\left(N_{\mathrm{H}_{\mathrm{I}}}=10^{13.15} \mathrm{~cm}^{-2}\right) \mathrm{H}_{\mathrm{I}} \mathrm{Ly} \alpha$ in COS, with a Doppler parameter of $b=46 \pm 8 \mathrm{~km} \mathrm{~s}^{-1}$. Correcting for the empirically derived $b / b_{\text {th }}$ ratio of $\simeq 1.2$ (Danforth et al. $2010 \mathrm{~b})$, gives a thermal width of $b_{\text {th }}=38_{-7}^{+16} \mathrm{~km} \mathrm{~s}^{-1}$, implying $T=(0.6-1.7) \times 10^{5} \mathrm{~K}$, consistent with either shock-heated WHIM or low-density purely photoionized IGM. However, we note that, given the relatively low-temperature derived from the $\mathrm{H}$ I Doppler parameter, the column density of this H I component is probably too low to produce all the $\mathrm{CV}$ and $\mathrm{C}$ VI detected in the LETG, even assuming solar abundances. Therefore, if our 


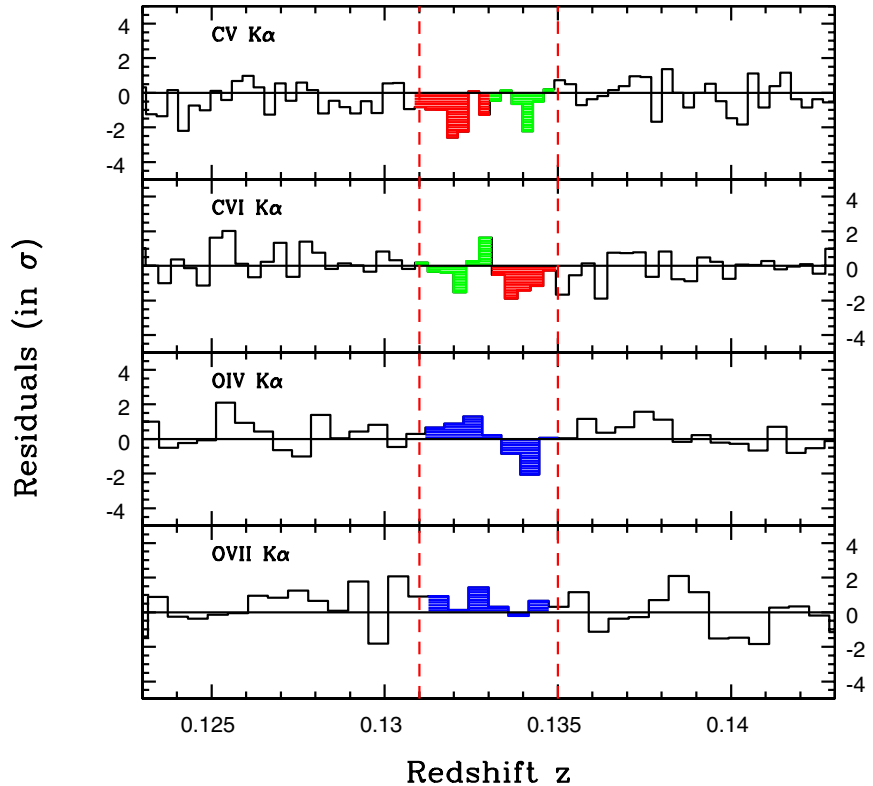

Figure 7. Four portions of the LETG spectrum of 1 ES $1553+113$, centered around the X-ray redshift of the $\mathrm{CV}-\mathrm{C}$ VI complex $(z=0.131-0.135)$, for C v K $\alpha$ (top panel), C vI K $\alpha$ (second panel), O IV K $\alpha$ (third panel), and O VII K $\alpha$ (bottom panel). Detected lines are filled in red, lines hinted in the data are filled in green, while non-detections have the spectral bins filled in blue. The two vertical dashed red lines delimit the X-ray redshift interval of the system.

(A color version of this figure is available in the online journal.)

identification is correct, the $\mathrm{C}$ V-C VI system cannot be entirely and directly associated with the $\mathrm{H}$ I Ly $\alpha$ in COS. Possibly some of the detected $\mathrm{CV}$ and $\mathrm{C}$ VI must be associated with an even broader and shallower $\mathrm{H}$ i not detectable at the sensitivity of the current COS spectrum.

To further investigate this possible identification, we visually inspected the portions of the LETG spectrum where the tentatively detected $\mathrm{C}$ V and $\mathrm{C}$ VI $\mathrm{K} \alpha$ transitions, as well as other, similar ionization associated lines of $\mathrm{O}$ and $\mathrm{C}$ would fall. Figure 7 shows four portions of the LETG spectrum of 1ES $1553+113$ centered around the $z=0.131-0.135$ interval, for the $\mathrm{C} v \mathrm{~K} \alpha$ (top panel), $\mathrm{C}$ VI K $\alpha$ (second panel), O IV K $\alpha$ (third panel), and $\mathrm{O}$ vII $\mathrm{K} \alpha$ (bottom panel) transitions. Interestingly, the complex profiles of the $\mathrm{C} \mathrm{V}$ and $\mathrm{C}$ VI $\mathrm{K} \alpha$ lines, suggest the presence of a double component (red and green filled bins in the top and second panels of Figure 7). No other associated X-ray line is hinted by the data (possibly with the exception of a small deficit of counts at one of the two components of OIV: third panel).

We conclude that the $\mathrm{Cv} / \mathrm{C}$ VI $\mathrm{K} \alpha$ complex at an average redshift of $\left\langle z_{X}\right\rangle=0.133 \pm 0.002$ (Figure 7) is the most likely identification for these two LETG line complexes (Table 1; Figure 1, green line; and Figure 7, top two panels), possibly produced in a multi-phase, multi-component WHIM filament together with the spatially associated BLA absorber.

\subsubsection{The $z_{X}=0.184-0.191$ Double-C V}

The line at $\lambda=47.68 \AA(3.6 \sigma)$ can only be identified as $\mathrm{C} v \mathrm{~K} \alpha$. No other ion of $\mathrm{C}$ can be associated with any metal and/or $\mathrm{HI}$ absorber in COS. Instead, the redshifts of the $\mathrm{Cv} \mathrm{K} \alpha$ identification, $z_{X}=0.184 \pm 0.001$ is close, but only marginally consistent, with the short-wavelength extreme of a narrow redshift interval, $z_{\mathrm{FUV}}=0.18640-0.18989$, where a rare triple-H I system is found in COS. No O VI is detected at $z_{\mathrm{FUV}}=0.18640$, while both the $z_{\mathrm{FUV}}=0.18773$ and the

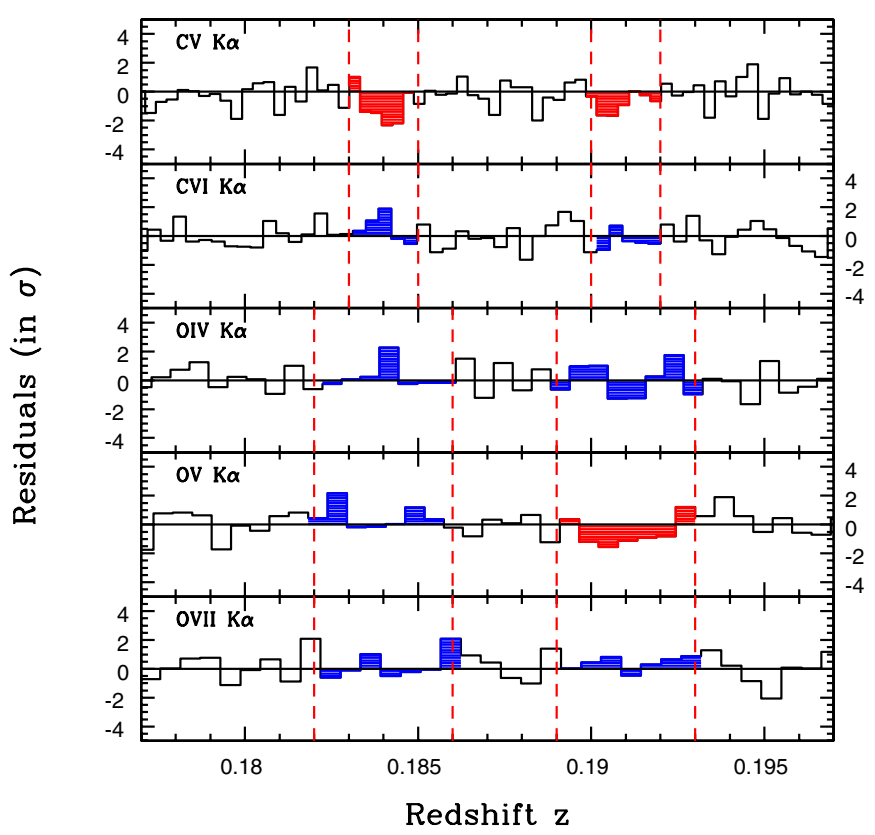

Figure 8. Five portions of the LETG spectrum of 1 ES $1553+113$ in the $z_{X}=0.175-0.195$ interval, for the $\mathrm{C} \mathrm{V} \mathrm{K} \alpha$ (top panel), the $\mathrm{C}$ VI K $\alpha$ (second panel), the O IV K $\alpha$ (third panel), the $\mathrm{Ov} \mathrm{K} \alpha$ (fourth panel), and the O VII K $\alpha$ (bottom panel) transitions. Detected lines are filled in red. Non-detections have the spectral bins filled in blue. The two pairs of vertical dashed red lines delimit the X-ray redshift intervals of the $\mathrm{C} v \mathrm{~K} \alpha$ identifications.

(A color version of this figure is available in the online journal.)

$z_{\mathrm{FUV}}=0.18989$ absorbers have associated O VI absorption, with the central system (the one with higher column density) showing also N v, C III, and Si II absorption (Danforth et al. 2010a). We re-analyzed these three absorbers in the currently available HST-COS spectrum of 1ES $1553+113$ and measured the Doppler parameters of the lines detected from the two H I-metal systems at $z_{\mathrm{FUV}}=0.18773$ and $z_{\mathrm{FUV}}=0.18989$. From these, we derive the thermal H i Doppler parameters of these two systems (by correcting for the average $b / b_{\text {therm }}=1 / 2$ value): $b_{\mathrm{HI}}^{\text {therm }}=27 \pm 10 \mathrm{~km} \mathrm{~s}^{-1}$ and $b_{\mathrm{HI}}^{\text {therm }}=58 \pm 4 \mathrm{~km} \mathrm{~s}^{-1}$, respectively. Clearly, the $\mathrm{HI}$-metal system at $z_{\mathrm{FUV}}=0.18773$ is imprinted by photoionized gas, while the $z_{\mathrm{FUV}}=0.18989$ $\mathrm{H}$ I-O VI absorber is a WHIM system with $\log T=5.3 \pm 0.1$.

Interestingly, in the LETG we also marginally detect $(2.2 \sigma)$ a line-like absorption feature at $\lambda=47.94 \AA$. Also for this line, if real, the only possible identification, in association with a COS $\mathrm{HI}$ and/or metal system, is $\mathrm{Cv} \mathrm{K} \alpha$ at $z_{X}=0.191 \pm 0.001$, consistent with the long-wavelength extreme of the COS tripleH I-metal system, where a relatively strong BLA $\left(\log N_{\mathrm{H}_{\mathrm{I}}}=\right.$ $13.565 \pm 0.017)$ is present. Figure 8 shows five portions of the LETG spectrum of 1 ES $1553+113$ in the $z_{X}=0.175-0.195$ interval, for the $\mathrm{C} v \mathrm{~K} \alpha$ (top panel), the $\mathrm{C}$ VI $\mathrm{K} \alpha$ (second panel), the $\mathrm{O}$ IV $\mathrm{K} \alpha$ (third panel), the $\mathrm{O} v \mathrm{~K} \alpha$ (fourth panel), and the $\mathrm{O}$ vII $\mathrm{K} \alpha$ (bottom panel) transitions. No associated X-ray lines is hinted in the data for the tentative $\mathrm{Cv} \mathrm{K} \alpha$ identification at $z=0.184 \pm 0.001$. The situation is different for the second tentative $\mathrm{C} v \mathrm{~K} \alpha$ identification at $z=0.191 \pm 0.001$ (the higher redshift boundary of the triple-H I-metal system detected in COS, where a BLA is present). For this system the $\mathrm{Ov} \mathrm{K} \alpha$ transition is identifiable with the lowest significance $(1.7 \sigma)$ line-like absorption feature identified by our scanning routine and subsequently confirmed by a Gaussian fit in the LETG, at $\lambda=26.65 \AA$. 
We thus speculate that these LETG lines at $\lambda=47.68 \AA$ $(3.6 \sigma), \lambda=47.94 \AA(2.2 \sigma)$ ), and $\lambda=26.65 \AA$ (only $1.7 \sigma)$, may be the high-ionization $\mathrm{C} v \mathrm{~K} \alpha\left(z_{X}=0.184\right.$ and $\left.z_{X}=0.191\right)$ and $\mathrm{O} \vee \mathrm{K} \alpha\left(z_{X}=0.191\right)$ counterparts (Table 1; Figure 8, top panel, red bins) of the interesting, likely multi-phase, triple-H Imetal system detected in COS, possibly witnessing the on-going interplay between nearby galaxy outflows and a WHIM filament at $z_{\mathrm{FUV}}=0.188989$, where a $\mathrm{C} \mathrm{V}-\mathrm{O} \mathrm{V}-\mathrm{BLA}$ system is present.

\subsubsection{The $z_{X}=0.041 \pm 0.02$ WHIM System}

Finally, the marginal detection at $\lambda=22.47 \AA(2.3 \sigma)$ can only be redshifted $\mathrm{O} v$ or higher. The $\mathrm{O} \vee \mathrm{K} \alpha$ transition would be redshifted to $z_{X}=0.005 \pm 0.002$. There is an NLA present in $\mathrm{COS}$ at $z_{\mathrm{FUV}}=0.00717$, i.e., marginally consistent with the $\mathrm{X}$-ray redshift. However, in photoionized gas $\mathrm{O} v$ shows fractional abundances similar to O vI. No O VI absorption is seen at this redshift either in the FUSE spectrum of 1ES $1553+113$ or in its LETG spectrum. The $\mathrm{O}$ vi $\mathrm{K} \alpha$ transition would be redshifted to $z_{X}=0.020 \pm 0.002$. No H I Ly $\alpha$ or O vi $\left(1 s^{2} 2 s \rightarrow 1 s^{2} 2 p\right)$ is seen at a consistent redshifts in COS or FUSE. Finally, the $\mathrm{O}$ VII and $\mathrm{O}$ VIII $\mathrm{K} \alpha$ transitions, would be redshifted to $z_{X}=$ $0.041 \pm 0.002$ and $z_{X}=0.185 \pm 0.002$. These two redshifts are both consistent with either the broadest $\left(b_{\mathrm{HI}}=73 \pm 7 \mathrm{~km} \mathrm{~s}^{-1}\right.$, corresponding to $\log T=5.3-5.6 \mathrm{~K})$ and strongest $(\mathrm{EW}(\mathrm{BLA})=$ $135 \pm 14 \mathrm{~m} \AA$ ) BLA detected in the published COS spectrum, at $z_{\mathrm{FUV}}=0.04281$, or the redshift range of the unusual tripleH I-metal system at $z_{\mathrm{FUV}}=0.18640-0.18989$, respectively. However, while a possible double-C v counterpart to the triple$\mathrm{H} \mathrm{I-metal} \mathrm{system} \mathrm{seen} \mathrm{in} \mathrm{COS} \mathrm{is} \mathrm{possibly} \mathrm{detected} \mathrm{in} \mathrm{the} \mathrm{LETG}$ (Section 4.1.5), no O VII counterpart is detected. So, it appears unlikely that the line at $\lambda=22.47 \AA$ is O VIII associated with the triple-H I-metal system.

For this marginal LETG detection we therefore favor the $z_{X}=0.041 \mathrm{O}$ VII-BLA association (Table 1, Columns 3-5 and Figure 1 magenta line), and tentatively identify this association with an intervening WHIM system at the COS-detected BLA signpost (though an O VIII-triple-H I association cannot be ruled out). No other associated X-ray line is hinted in the data (Figure 9).

We stress that this is the weakest system for which we propose an identification in association with a COS system (only one LETG line, at $2.3 \sigma$ ). However, given the nature of this association (O VII-BLA), in principle probing the majority of the baryons in the WHIM, a confirmation of this putative $\mathrm{O}$ VII $\mathrm{K} \alpha$ detection at high statistical significance is highly desirable.

\subsection{On the Statistical Significance of the $X$-ray Absorption Lines}

The statistical significances of the X-ray absorption lines reported in Table 1 (second column) are single-line statistical significances. That is the ratio between the line flux, and its $1 \sigma$ lower-bound uncertainty. For those lines not identifiable with $z \simeq 0$ transitions imprinted by Galactic or circumgalactic gas, these significances overestimate the true absorption-line statistical significances, because they derive from a number of redshift trials. This number of redshift trials, depends on the wavelength of the transition, the spectrometer resolution, and the available path length over which the blind search is performed: $N=\left(\lambda_{0} \times \Delta z\right) / \Delta \lambda_{\text {spec }}$, where $\lambda_{0}$ is the rest-frame wavelength of the given transition, and $\Delta \lambda_{\text {spec }}$ the spectrometer LSF FWHM. In our case, $\Delta z=0.4$ and $\Delta \lambda_{\text {spec }}=50 \mathrm{~m} \AA$. So, the number of independent LETG resolution elements is 173 for the $\mathrm{O}$ vII $\mathrm{K} \alpha$ and 322 for the $\mathrm{C} v \mathrm{~K} \alpha$. In such conditions, the true

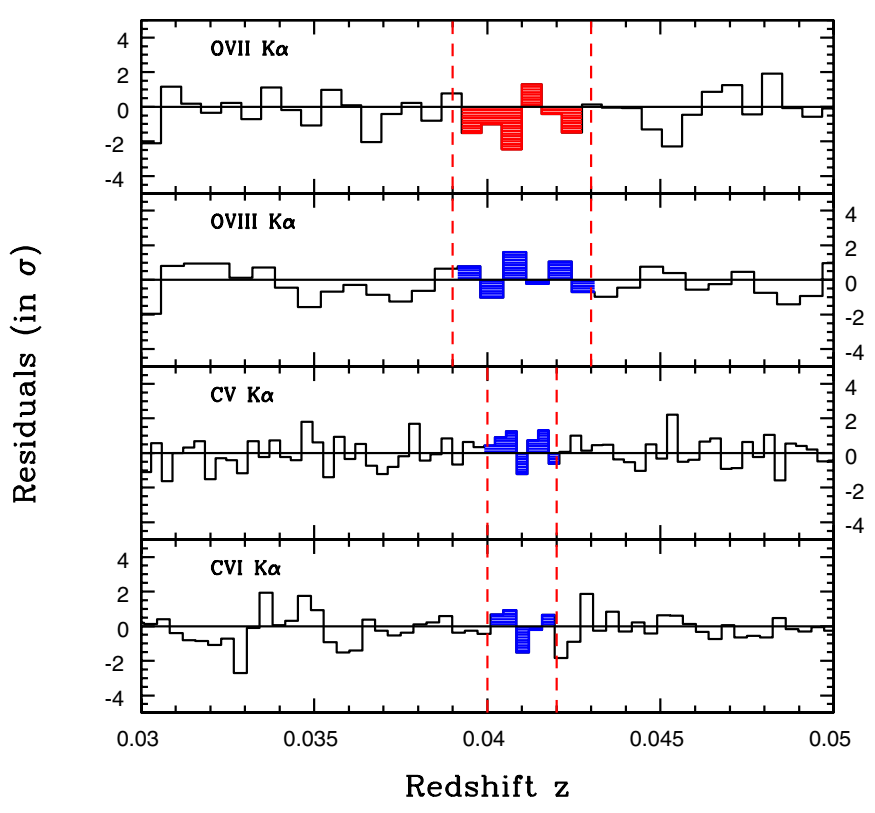

Figure 9. Four portions of the LETG spectrum of 1ES $1553+113$ in the $z_{X}=0.03-0.05$ interval, for the $\mathrm{O}$ vII $\mathrm{K} \alpha$ (top panel), the $\mathrm{O}$ vIII $\mathrm{K} \alpha$ (second panel), the $\mathrm{C}$ V K $\alpha$ (third panel), and the $\mathrm{C}$ VI $\mathrm{K} \alpha$ (bottom panel) transitions. Detected lines are filled in red. Non-detections have the spectral bins filled in blue. The two vertical dashed red lines delimit the X-ray redshift intervals of the $\mathrm{O}$ vII $\mathrm{K} \alpha$ identifications.

(A color version of this figure is available in the online journal.)

statistical significance (i.e., after correcting for the unknown line position) of a line blindly detected at a "single-line" statistical significance of $4 \sigma$ is only $2.6 \sigma$ and $2.4 \sigma$, for O VII $\mathrm{K} \alpha$ and $\mathrm{C} \vee \mathrm{K} \alpha$, respectively.

However, if an independent (and physically self-consistent) redshift prior is known (O vI or BLA in COS), then the line identification can be considered "secure" and therefore the single-line statistical significance provides a reliable estimate of the true statistical significance of the line. This is true for five out of the eight intervening lines: (1) the highest statistical significance $z_{X}=0.312 \mathrm{C} v$ and $\mathrm{C}$ vI lines, at redshift consistent with that of a strong BLA-O VI system with the right temperature to produce $\mathrm{Cv}$; (2) the $z_{X}=0.237 \mathrm{C} \mathrm{v}$ line, at redshift consistent with that of a shallow BLA complex with the right temperature to produce $\mathrm{Cv}$; (3) the putative $z_{X}=0.191 \mathrm{C} \mathrm{v}-\mathrm{O}$ v lines, at redshifts consistent with that of a BLA at $z_{\mathrm{FUV}}=0.18989$ in COS; and (4) the speculative $z_{X}=0.041 \mathrm{O}$ VII absorber, at redshift consistent with that of a strong BLA whose thermal width is appropriate for the production of $\mathrm{O}$ VII.

The remaining three lines are identified as $\mathrm{Cv} \mathrm{K} \alpha$ at $z_{X}=$ 0.184 (close to the lower boundary of a rare triple $\mathrm{H}$ I-metal system detected in COS), and C v-C vI K $\alpha$ at $\left\langle z_{X}\right\rangle=0.133$, a redshift consistent with that of a BLA whose column density, however, is probably too low to produce the entire $\mathrm{C} \mathrm{V}$ and $\mathrm{C}$ VI absorption (given the relatively low temperature derived for this BLA). For the putative $\mathrm{Cv} \mathrm{K} \alpha$ line at $z_{X}=0.184 \pm 0.001$, its single-line significance is $3.6 \sigma$, which corresponds to a probability of chance detection of $3.5 \times 10^{-4}$. Allowing for the number of redshift trials, the probability of chance detection of this line rises to 0.056 , a Gaussian-equivalent significance of $1.9 \sigma$. For the $\mathrm{C}$ V $-\mathrm{C}$ VI system at $\left\langle z_{X}\right\rangle=0.133$, the presence of two lines poses a bound on the number of redshift trials for the line detected at lower statistical significance and makes its measured single-line statistical significances close to the true statistical significances. These two lines are detected at 
single-line significances of $3.8 \sigma$ and $2.7 \sigma$, corresponding to probabilities of chance detections of $1.5 \times 10^{-4}$ and $7 \times 10^{-3}$, respectively. Allowing for the number of redshift trials, the probability of chance detection of the strongest of these two lines rises to 0.024, a Gaussian-equivalent significance of $2.3 \sigma$. However, considering this as a prior for the second line, the probability of having by chance both lines (independently) is the product of the two probabilities: $P=0.024 \times 0.007=$ $1.7 \times 10^{-4}$, corresponding to a Gaussian-equivalent significance of $3.8 \sigma$.

\section{CONCLUSIONS}

We report on the detection of six absorption lines and five additional features associated with either other X-ray lines detected at higher significance and/or FUV signposts, in the $500 \mathrm{ks}$ Chandra-LETG spectrum of the bright $\left(F_{0.1-2}=\right.$ $1.2 \mathrm{mCrab})$ and distant $(z \geqslant 0.4)$ blazar 1ES $1553+113$. The six detections have single-line statistical significances between $3.6 \sigma$ and $4.1 \sigma$, while the five additional associations are detected with single-line statistical significances of $2.2 \sigma-2.8 \sigma$.

We tentatively identify these absorption lines as belonging to six different systems: the Galaxy cold ISM, and five intervening warm-hot IGM filaments.

Three out of the five intervening absorption systems show multiple-ion associations in the X-rays, have BLA (and O vi for the system at $z_{X}=0.312$ ) counterparts at consistent redshifts in the HST-COS spectrum of 1ES $1553+113$ and have true X-ray statistical significances of $5.8 \sigma\left(z_{X}=0.312 ; 6.3 \sigma\right.$ if the lowsignificance $\mathrm{O} v$ and $\mathrm{C} v \mathrm{~K} \beta$ associations are considered), $3.9 \sigma$ $\left(z_{X}=0.237\right)$ and $3.8 \sigma\left(\left\langle z_{X}\right\rangle=0.133\right)$. For these three systems, the COS counterparts are also physically consistent with the ionization degree and temperature of the X-ray absorbers in a shock-heated gas scenario. However, for the $\left\langle z_{X}\right\rangle=0.133$ system, given the temperature implied by the BLA width, the $\mathrm{H}$ I column density is probably too low to produce all the detected $\mathrm{C}$ v and $\mathrm{C}$ vi absorption.

The two remaining proposed identifications are X-ray singleion tentative detections (with the exception of the $\mathrm{C} v-\mathrm{BLA}-\mathrm{O} \mathrm{VI}$ $z_{X}=0.191$ system, where $\mathrm{O} v$ may also be present), both with a possible, physically consistent, COS counterpart: (1) the double $\mathrm{C} v \mathrm{~K} \alpha$ system at $z_{X}=0.184(3.6 \sigma)$ and $z_{X}=0.191(2.2 \sigma$, plus $\mathrm{O} v \mathrm{~K} \alpha, 1.7 \sigma$ ), both with $\mathrm{H}$ I (BLA-O vi for the putative $\mathrm{C} v-\mathrm{O} v$ at $\left.z_{x}=0.191\right)$ counterparts; and (2) the possible O vII $\mathrm{K} \alpha$ absorber at $z_{X}=0.041(2.3 \sigma)$ with a BLA counterpart.

Here, we summarize our identifications as follows.

1. Galactic ISM. We detect atomic O I (and possibly an excess of ISM oxygen in compound forms, over the instrumental molecular $\mathrm{O}$ I), as well as $\mathrm{C}_{\mathrm{II}}$ at $z \simeq 0$, and associate these absorbers with the cold ISM of our Galaxy along the line of sight to 1ES $1553+113$.

2. Intervening system $I$. This is the highest significance LETG system, with two lines detected at single-line statistical significances of $4.1 \sigma$ each. We identify these two lines with $\mathrm{CV}$ and $\mathrm{C}$ VI absorption at $z_{X}=0.312 \pm 0.001$, possibly imprinted by an intervening WHIM filament, as further suggested by our a posteriori discovery of an associated strong BLA and OVI counterpart in the COS spectrum, with the right temperature (i.e., line Doppler parameters) and strength. Associated $\mathrm{O} v$ and $\mathrm{Cv} \mathrm{K} \beta$ absorption are also possibly hinted by the X-ray data. The combined (in quadrature) statistical significance of this system in the LETG ( $\mathrm{C} \mathrm{v}$ and $\mathrm{C} \mathrm{v} \mathrm{K \alpha}$ lines only) is $5.8 \sigma$. If the association with $\mathrm{Ov} \mathrm{K} \alpha$ and $\mathrm{CVI} \mathrm{K} \beta$ is considered, the statistical significance of the system is $6.3 \sigma$.

3. Intervening System II. We identify $\mathrm{C}$ v absorption at $z_{X}=$ $0.237 \pm 0.001$, consistent with the redshift of a structured BLA complex that we discovered a posteriori in the currently available COS spectrum of 1ES 1553+113. Given the presence of a physically self-consistent redshift prior (the BLA complex in COS), the true statistical significance of this line in the LETG is of $3.9 \sigma$.

4. Intervening system III. We identify a C v-C VI-BLA system at $\left\langle z_{X}\right\rangle=0.133 \pm 0.002$, and associate this absorber with an intervening warm WHIM filament, where only part of the X-ray absorption is physically related to the detected BLA. The statistical significance of this system in the LETG (after properly accounting for the number of redshift trials) is $3.8 \sigma$.

5. Tentative intervening system $I V$. We identify two $\mathrm{C} \mathrm{v} \mathrm{K \alpha}$ absorbers at redshifts $z_{X}=0.184 \pm 0.001$ and $z_{X}=0.191 \pm$ 0.001 , where also $\mathrm{O} v \mathrm{~K} \alpha$ is tentatively detected, adjacent to the redshifts of the extremes of a narrow redshift interval containing a rare triple-H I-metal absorption system. One of these three $\mathrm{H}$ I absorber is a BLA-O vi pair, with redshift and temperature fully consistent with that of the $\mathrm{C} v-\mathrm{O} v$ absorber at $z_{X}=0.191 \pm 0.001$. We speculate that the $\mathrm{X}$-ray $\mathrm{Cv}-\mathrm{Ov}$ absorbers trace the same multi-phase medium traced by the triple-H I-metal system in COS, possibly representing the result of the interplay between galaxy winds and a WHIM filament at $z_{X}=0.191 \pm 0.001$. The $\mathrm{C}$ v line at $z_{X}=0.184 \pm 0.001$ is detected at single-line statistical significance of $3.6 \sigma$ (a true statistical significance of only $1.9 \sigma$, after allowing for the number of redshift trials), while the $\mathrm{C}$ V-BLA-O VI system at $z_{X}=0.191 \pm 0.001$ is detected at a true significance of $2.2 \sigma(2.8 \sigma$ if the $\mathrm{O} v \mathrm{~K} \alpha$ association is considered).

6. Tentative intervening system $V$. We tentatively identify an O VII-BLA system at $z_{X}=0.041 \pm 0.002$ and associate this absorber with an intervening hot WHIM filament. This is the weakest system for which we report a possible identification, with only one X-ray line at a single-line statistical significance of $2.3 \sigma$, but with a possible redshift prior identified in the broadest and strongest BLA reported in the published COS spectrum.

This moderate quality $500 \mathrm{ks}$ Chandra-LETG spectrum of the, by far, brightest steady soft X-ray target in the $z>0.4$ sky, strongly hints at the existence of possible X-ray metal WHIM counterparts to genuine gaseous signposts, previously secured with the HST-COS (or vice versa), and so demonstrates the efficiency of this observational strategy and the power of the X-ray-FUV synergy for WHIM studies. Moreover, these results suggest that the gas may be often found to be multi-phase, possibly indicating that the IGM structure is richer than typically found in hydrodynamical simulations. Longer integrations with both the available high-resolution soft X-ray spectrometers (the Chandra-LETG and the XMM-Newton-RGSs) are needed to confirm at high statistical significances and with two independent measurements these tentative identifications and associations (especially for the three lowest statistical significance associations), and to conduct a sensitive exploration of even hotter WHIM systems along this line of sight.

We thank Ehud Behar for providing wavelength, oscillator strengths, and transition probabilities of inner shell transitions of $\mathrm{C}, \mathrm{O}$, and Ne. F.N. acknowledges support from 
Chandra Grant GO1-12174X, and from INAF-ASI ADAE Grant 1.05.04.13.15. M.S. and C.D. acknowledge support from COS Grant NNX08AC14G to the University of Colorado, from NASA. X.B. acknowledges support by the Spanish Ministry of Economy and Competitiveness through grant AYA2010-21490C02-01.

\section{REFERENCES}

Asplund, M., Grevesse, N., Sauval, A. J., \& Scott, P. 2009, ARA\&A, 47, 481 Bar-Shalom, A., Klapish, M., \& Oreg, J. 2001, JQSRT, 71, 169

Behar, E., \& Netzer, H. 2002, ApJ, 570, 165

Bennett, C. L., Halpern, M., Hinshaw, G., et al. 2003, ApJS, 148, 1

Bertone, S., Schaye, J., Dalla Vecchia, C., et al. 2010, MNRAS, 407, 544

Branchini, E., Ursino, E., Corsi, A., et al. 2009, ApJ, 697, 328

Brinkman, A. C., Gunsing, C. J. T., Kaastra, J. S., et al. 2000, ApJL, 530, L111

Buote, D. A., Zappacosta, L., Fang, T., et al. 2009, ApJ, 695, 1351

Cen, R., \& Fang, T. 2006, ApJ, 650, 573

Cen, R., \& Ostriker, J. P. 2006, ApJ, 650, 560

Danforth, C. W., Keeney, B. A., Stocke, J. T., Shull, J. M., \& Yao, Y. 2010a, ApJ, 720,976

Danforth, C. W., \& Shull, J. M. 2008, ApJ, 679, 194

Danforth, C. W., Stocke, J. T., Keeney, B. A., et al. 2011, ApJ, 743, 18

Danforth, C. W., Stocke, J. T., \& Shull, J. M. 2010b, ApJ, 720, 976

den Herder, J. W., Brinkman, A. C., Kahn, S. M., et al. 2001, A\&A, 365, L7

Fang, T., Buote, D. A., Humphrey, P. J., et al. 2010, ApJ, 714, 1715

Gupta, A., Mathur, S., Krongold, Y., Nicastro, F., \& Galeazzi, M. 2012, ApJL, 756, L8

Juett, A. M., Schulz, N. S., \& Chakrabarty, D. 2004, ApJ, 612, 308

Kaastra, J. S., de Vries, C. P., Steenbrugge, K. C., et al. 2011, A\&A, 534, 37

Kaastra, J. S., Werner, N., den Herder, J. W. A., et al. 2006, ApJ, 652, 189
Kalberla, P. M. W., Burton, W. B., Hartmann, D., et al. 2005, A\&A, 440, 775 Keshet, U., Kushnir, D., Loeb, A., \& Waman, E. 2012, arXiv:1210.1574

Kirkman, D., Tytler, D., Suzuki, N., O’Meara, J. M., \& Lubin, D. 2003, ApJS, 149, 1

Mathur, S., Weinberg, D. H., \& Chen, X. 2003, ApJ, 582, 82

Murray, S. S., \& Chappell, J. H. 1996, Proc. SPIE, 597, 279

Nicastro, F., Krongold, Y., Fields, D., et al. 2010, ApJ, 715, 854

Nicastro, F., Mathur, S., \& Elvis, M. 2008, Sci, 319, 55

Nicastro, F., Mathur, S., Elvis, M., et al. 2005a, Natur, 433, 495

Nicastro, F., Mathur, S., Elvis, M., et al. 2005b, ApJ, 629, 700

Nicastro, F., Zezas, A., Drake, J., et al. 2002, ApJ, 573, 157

Rasmussen, A. P., Kahn, S. M., Paerels, F., et al. 2007, ApJ, 656, 129

Rauch, M. 1998, ARA\&A, 36, 267

Schulz, N. S., Juett, A., Chakrabarty, D., \& Canizares, C. R. 2003, AN, 324,166

Shull, J. M., Smith, B. D., \& Danforth, C. W. 2012, ApJ, 759, 23

Smith, B. D., Hallman, E. J., Shull, J. M., \& O'Shea, B. W. 2011, ApJ, 731, 6

Spergel, D. N., Bean, R., Doré, O., et al. 2007, ApJS, 170, 377

Takei, Y., Fujimoto, R., \& Mitsuda, K. 2002, ApJ, 581, 307

Tornatore, L., Borgani, S., Viel, M., \& Springel, V. 2010, MNRAS, 402, 1911

Treves, A., Falomo, R., \& Uslenghi, M. 2007, A\&A, 473, L17

Verner, D. A., Verner, E. M., \& Ferland, G. J. 1996, AtPhy, 4003

Weinberg, D. H., Miralda-Escude, J., Hernquist, L., \& Katz, N. 1997, ApJ, 490, 564

Williams, R. J., Mathur, S., Nicastro, F., \& Elvis, M. 2006, ApJL, 624, L95

Williams, R. J., Mulchaey, J. S., \& Kollmeier, J. A. 2012, ApJL, 762, L10

Williams, R. J., Mulchaey, J. S., Kollmeier, J. A., \& Cox, T. J. 2010, ApJL, 724, L25

Yao, Y., Shull, J. M., Wang, Q. D., \& Cash, W. 2012, ApJ, 746, 166

Yoshikawa, K., Yamasaki, N. Y., Suto, Y., et al. 2003, PASJ, 55, 879

Zappacosta, L., Nicastro, F., Krongold, Y., \& Maiolini, R. 2012, ApJ, 753, 137

Zappacosta, L., Nicastro, F., Maiolino, R., et al. 2010, ApJ, 717, 74 